\title{
Synthetic biology of cyanobacteria: unique challenges and opportunities
}

\section{Bertram M. Berla ${ }^{1}$, Rajib Saha ${ }^{2}$, Cheryl M. Immethun ${ }^{1}$, Costas D. Maranas ${ }^{2}$, Tae Seok Moon ${ }^{1}$ and Himadri B. Pakrasi ${ }^{1,3 *}$}

${ }^{1}$ Department of Energy, Environmental, and Chemical Engineering, Washington University, St. Louis, MO, USA

${ }^{2}$ Department of Chemical Engineering, Pennsylvania State University, University Park, PA, USA

${ }^{3}$ Department of Biology, Washington University in St. Louis, St. Louis, MO, USA

Edited by:

Aindrila Mukhopadhyay, Lawrence Berkeley National Berkeley, USA

\section{Reviewed by:}

Dong-Woo Lee, Kyungpook National

University, South Korea

Patrick Hallenbeck, University of

Montreal, Canada

\section{*Correspondence:}

Himadri B. Pakrasi, Department of Biology, Washington University in St. Louis, Campus Box 1095, One Brookings Drive, St. Louis, MO 63130-4899, USA

e-mail: pakrasi@wustl.edu
Photosynthetic organisms, and especially cyanobacteria, hold great promise as sources of renewably-produced fuels, bulk and specialty chemicals, and nutritional products. Synthetic biology tools can help unlock cyanobacteria's potential for these functions, but unfortunately tool development for these organisms has lagged behind that for $S$. cerevisiae and $E$. coli. While these organisms may in many cases be more difficult to work with as "chassis" strains for synthetic biology than certain heterotrophs, the unique advantages of autotrophs in biotechnology applications as well as the scientific importance of improved understanding of photosynthesis warrant the development of these systems into something akin to a "green E. coli." In this review, we highlight unique challenges and opportunities for development of synthetic biology approaches in cyanobacteria. We review classical and recently developed methods for constructing targeted mutants in various cyanobacterial strains, and offer perspective on what genetic tools might most greatly expand the ability to engineer new functions in such strains. Similarly, we review what genetic parts are most needed for the development of cyanobacterial synthetic biology. Finally, we highlight recent methods to construct genome-scale models of cyanobacterial metabolism and to use those models to measure properties of autotrophic metabolism. Throughout this paper, we discuss some of the unique challenges of a diurnal, autotrophic lifestyle along with how the development of synthetic biology and biotechnology in cyanobacteria must fit within those constraints.

Keywords: cyanobacteria, synthetic biology, systems biology, biofuel, flux balance analysis, metabolic flux analysis

\section{INTRODUCTION}

Cyanobacteria have garnered a great deal of attention recently as biofuel-producing organisms. Their key advantage over other bacteria is their ability to use photosynthesis to capture energy from sunlight and convert $\mathrm{CO}_{2}$ into products of interest. As compared with eukaryotic algae and plants, cyanobacteria are much easier to manipulate genetically and grow much faster. They have been engineered to produce a wide and ever-expanding range of products including fatty acids, long-chain alcohols, alkanes, ethylene, polyhydroxybutyrate, 2,3-Butanediol, ethanol, and hydrogen. These processes have been reviewed recently (Gronenberg et al., 2013) and will not be covered in detail in this review. Rather, we will look toward how the techniques of the emerging field of synthetic biology might bear fruit in improving the output of such engineered strains. Due to the low price of commodity goods like fuels and platform chemicals, it is critical to maximize the productivity of engineered strains to make them economically competitive. We believe that the tools of synthetic biology can help with this challenge.

Specifically, this review will cover systems, parts, and methods of analysis for synthetic biology. Synthetic biology requires a well-characterized host or "chassis" strain that can be genetically manipulated with ease and predictability. Ideally, the host should grow quickly and tolerate a range of environmental conditions. The host should be simple to cultivate using readily available laboratory equipment and inexpensive growth media. Simple, rapid, and high-throughput techniques should be available for procedures like DNA/RNA isolation, metabolomics, and proteomics. To achieve modular, "plug-and-play" modification of the host strain, its metabolism and regulatory systems must be wellcharacterized under a wide variety of relevant conditions. Since cyanobacterial biofuel production processes will need to use sunlight as an energy source to be economically and environmentally useful, the day/night cycle will be particularly relevant; the intermittent nature of this energy source will be a key engineering challenge. We will discuss which cyanobacterial chassis have been used and their relative merits and unique traits. Ultimately, the hope is that one of these strains might be developed to become a "green E. coli" for which a wide variety of genetic parts and systems are available for easy modification. Next, we will discuss the critical issue of how gene expression can be controlled in cyanobacteria. Compared with other systems, there are few examples of simple and effective controllable promoters in cyanobacteria. We will also discuss methods for analysis of gene expression using light-emitting reporters and for global analysis of metabolism using either constraint-based modeling or measurement of ${ }^{13} \mathrm{C}$ labeling. 


\section{GENETIC MODIFICATION OF CYANOBACTERIA}

Several strains of cyanobacteria are known which are readily amenable to genetic modification (See Table 1). Such modifications can be performed either in cis (through chromosome editing) or in trans (through plasmid addition) and synthetic biology experiments have used both approaches. We discuss advantages and disadvantages of each approach, as well as recent technical developments below. While even the best cyanobacterial model systems are still far from being a "green E. coli," many tools are already available and more are being developed. The future holds great promise for this field.

\section{GENETIC MODIFICATION IN CIS: CHROMOSOME EDITING}

Cis genetic modification is the most common approach in cyanobacterial synthetic biology. This approach takes advantage of the capability of many cyanobacterial strains for natural transformation and homologous recombination (see Table 1) to create insertion, deletion, or replacement mutations in cyanobacterial chromosomes. Traditionally, strains have been transformed with selectable markers linked to any sequence of interest and flanked by sequences homologous to any non-essential sequence on the chromosome (See Figure 1).

This strategy allows the creation of targeted mutations to the chromosome, but sometimes raises concerns about segregation in polyploid strains. However, once segregated, such mutations can be stable over long time periods even in the absence of selective pressure from added antibiotics (Liu et al., 2011; Wang et al., 2013). While such stability is desirable, systems that create major metabolic demand, by for example redirecting flux into biofuel-producing pathways, will face greater selective pressures for mutation or loss of heterologous genes.
Recently, several methods have been developed that allow the creation of markerless mutations in cyanobacterial chromosomes (Figure 1B). Two of these methods operate on a similar principle: First, a conditionally toxic gene is linked to an antibiotic resistance cassette and then inserted into the chromosome,

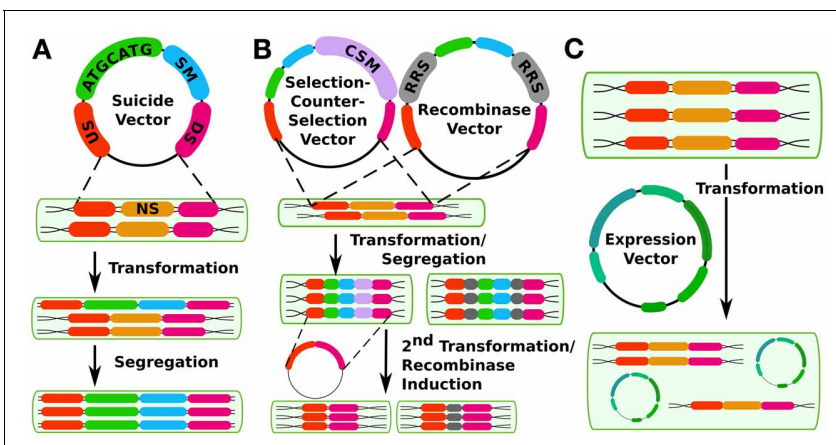

FIGURE 1 | Different methods for constructing cyanobacterial mutants (A) shows the traditional method using double homologous recombination to insert a suicide vector into the genome at a neutral site (NS, gold) with upstream (US, orange) and downstream (DS, magenta) flanking regions in the vector. The insert contains an arbitrary sequence of interest (ATGCATG, green) and a selectable marker (SM, blue). (B) shows two methods of creating markerless mutants, either by selection-counterselection or by using a recombinase system such as FLP/FRT, The counter-selection method's first step is the same as for the method in panel a, except that the insert also contains a counter-selectable marker (CSM, purple) such as $\operatorname{sac} B$. A second transformation is performed to create a markerless mutant. Alternatively, the insert can contain recombinase recognition sites (RRS, gray) that are controlled by an inducible recombinase at a second (or the same) site in the genome. While it erases the selectable marker, this method does leave a scar sequence behind. (C) shows genetic modification in trans via expression plasmids.

Table 1 | Model strains of cyanobacteria for synthetic biology.

\begin{tabular}{|c|c|c|c|c|c|c|c|}
\hline Strain & $\begin{array}{l}\text { Genetic } \\
\text { methods }\end{array}$ & $\begin{array}{l}\text { Ideal growth } \\
\text { temp (C) }\end{array}$ & $\begin{array}{l}\text { Doubling } \\
\text { time (h) }\end{array}$ & Metabolisms & $\begin{array}{l}\text { Genome-scale } \\
\text { models? }\end{array}$ & Notes & References \\
\hline $\begin{array}{l}\text { Synechocystis } \\
\text { sp. PCC } 6803\end{array}$ & $\begin{array}{l}\text { Conjugation, natural } \\
\text { transformation, Tn5 } \\
\text { mutagenesis, fusion } \\
\text { PCR }\end{array}$ & 30 & $6-12$ & $\begin{array}{l}\text { Mixotrophic, } \\
\text { autotrophic }\end{array}$ & Yes & $\begin{array}{l}\text { Extensive systems } \\
\text { biology datasets } \\
\text { are available }\end{array}$ & Heidorn et al., 2011 \\
\hline $\begin{array}{l}\text { Synechococcus } \\
\text { elongatus PCC } \\
7942\end{array}$ & $\begin{array}{l}\text { Conjugation, natural } \\
\text { Transformation, Tn5 } \\
\text { mutagenesis }\end{array}$ & 38 & $12-24$ & Autotrophic & No & $\begin{array}{l}\text { A model strain for } \\
\text { the study of } \\
\text { circadian clocks }\end{array}$ & Chen et al., 2012 \\
\hline $\begin{array}{l}\text { Synechococcus } \\
\text { sp. PCC } 7002\end{array}$ & $\begin{array}{l}\text { Conjugation, natural } \\
\text { transformation }\end{array}$ & 38 & 3.5 & $\begin{array}{l}\text { Mixotrophic, } \\
\text { autotrophic }\end{array}$ & Yes & $\begin{array}{l}\text { Among the } \\
\text { fastest-growing } \\
\text { strains known }\end{array}$ & Xu et al., 2011 \\
\hline $\begin{array}{l}\text { Anabaena } \\
\text { variabilis PCC } \\
7120\end{array}$ & $\begin{array}{l}\text { Conjugation, natural } \\
\text { transformation }\end{array}$ & 30 & $>24$ & $\begin{array}{l}\text { Mixotrophic, } \\
\text { autotrophic }\end{array}$ & No & $\begin{array}{l}\text { Nitrogen-fixing, } \\
\text { Filamentous }\end{array}$ & Zhang et al., 2007 \\
\hline $\begin{array}{l}\text { Leptolyngbya sp. } \\
\text { Strain BL0902 }\end{array}$ & $\begin{array}{l}\text { Conjugation, Tn5 } \\
\text { mutagenesis }\end{array}$ & 30 & $\sim 20$ & Autotrophic & No & $\begin{array}{l}\text { Filamentous, } \\
\text { Grows well in } \\
\text { outdoor } \\
\text { photo-bioreactors } \\
\text { in a broad range of } \\
\text { conditions }\end{array}$ & Taton et al., 2012 \\
\hline
\end{tabular}


with selection for antibiotic-resistant mutants. Next, a second transformation is carried out in which the resistance cassette and toxin gene are deleted, and markerless mutants are selected which have lost the toxic gene. This principle has been used in cyanobacteria with the B. subtilis levansucrase synthase gene $s a c B$, which confers sucrose sensitivity (Lagarde et al., 2000) as well as with E. coli $m a z F$, a general protein synthesis inhibitor expressed under a nickel-inducible promoter (Cheah et al., 2013). This latter system has advantages for cyanobacterial strains that are naturally sucrose-sensitive. Either method allows the reuse of a single selectable marker for making multiple successive changes to the chromosome. In addition to these methods, a third system operates on a similar principle - a cyanobacterial strain that is streptomycin resistant due to a mutation in the rps12 gene can be made streptomycin-sensitive by expressing a second heterologous copy of wild type rps12 linked to a kanamycin (or other antibiotic) resistance cassette as well as any sequence of interest. Streptomycin-resistant, kanamycin-sensitive markerless mutants can be recovered in a second transformation (Takahama et al., 2004). Although this method can also be used to make successive markerless mutants, it requires a background strain that is streptomycin-resistant due to an altered ribosome. Thus, it may not be an ideal method for synthetic biology studies that seek to draw conclusions about translation in wild-type systems. For the ability to transfer any translated genetic parts or parts involved in translation (such as ribosome binding sites) to other strains, this mutation could be problematic. A possible advantage of this system is that both selections are positive selections, whereas the $s a c B$ or $m a z F$ systems require a negative selection in their second transformation. Care must be taken to ensure that sucrose resistance is due to loss, as opposed to mutation, of the counter-selectable marker. Recombinase-based systems including Cre-LoxP [in Anabaena sp. PCC7120, (Zhang et al., 2007)] or FLP/FRT [in Synechocystis sp. PCC6803 and Synechococcus elongatus PCC7942, (Tan et al., 2013)] have also been used to engineer mutants that lack a selectable marker. However, these methods leave a scar sequence, meaning that the final chromosomal sequence is not completely user-specifiable and also that multiple mutations using this technique in the same cell line may potentially lead to undesirable crossover events or other unexpected results.

Until recently, it has been difficult to create mutants at high throughput in cyanobacterial strains, as transposon-based methods developed for use in other strains can work poorly in cyanobacterial hosts. However, libraries can be created in other strains and subsequently transferred to a cyanobacterial host via homologous recombination. A Tn7-based library containing $\sim 10,000$ lines was recently created to screen for strains with increased polyhydroxybutyrate (PHB) production (Tyo et al., 2009) and a similar approach has been taken for finding mutants in circadian clock function in Synechococcus 7942 (Holtman et al., 2005) and later extended to include insertions into nearly $90 \%$ of open reading frames in that strain (Chen et al., 2012). Chromosomal DNA fragments were first cloned into a plasmid library in E. coli and then the library was mutagenized with $\operatorname{Tn} 7$ before homologous recombination back into the cyanobacterial host strain. This could be an especially valuable approach for the validation of genome-scale models of cyanobacterial metabolism (see below).

\section{GENETIC MODIFICATION in trans: FOREIGN PLASMIDS}

Although transgene expression in cis is the most common approach in cyanobacterial research, genes are also routinely expressed in cyanobacteria in trans (Huang et al., 2010; Landry et al., 2012; Huang and Lindblad, 2013). In synthetic biology and metabolic engineering of other prokaryotes, this is by far the more common approach, and has led to such standardized approaches as "Bio-Brick" assembly in which standardized genetic "parts" such as promoters, ribosome binding sites, genes, and terminators can be readily swapped in and out of standard plasmids (http://partsregistry.org). This move toward standardization of genetic parts is a critical aim for synthetic biology, independent of the chassis organism or method of transformation. However, a limited number of plasmids are available for expression in cyanobacterial hosts. Plasmid assembly for expression in cis or in trans in cyanobacterial hosts has generally been performed in E. coli because of the longer growth times that would be associated with assembling vectors in cyanobacterial hosts (Figure 2A). This requires broad host range plasmids. However, with the rise of in vitro assembly methods such as SLIC (Li and Elledge, 2007), Gibson assembly (Gibson et al., 2009), CPEC (Quan and Tian, 2009), fusion PCR (Szewczyk et al., 2007), and Golden Gate (Engler and Marillonnet, 2011), this limitation may become less important over time (Figure 2B). These next-generation cloning methods have been reviewed elsewhere (Hilson et al., 2012) and will not be covered here. Fusion PCR has been used to construct linear DNA fragments for homologous recombination in cyanobacterial chromosomes (Nagarajan et al., 2011), but to our knowledge replicative vectors for cyanobacteria have so far not been constructed without the use of a helper heterotrophic strain. Techniques for in vivo assembly of plasmids that have been developed for yeast (Shao and Zhao, 2009) may be adaptable to cyanobacteria because of their facility for homologous recombination (Figure 2C). Such an improvement could greatly speed

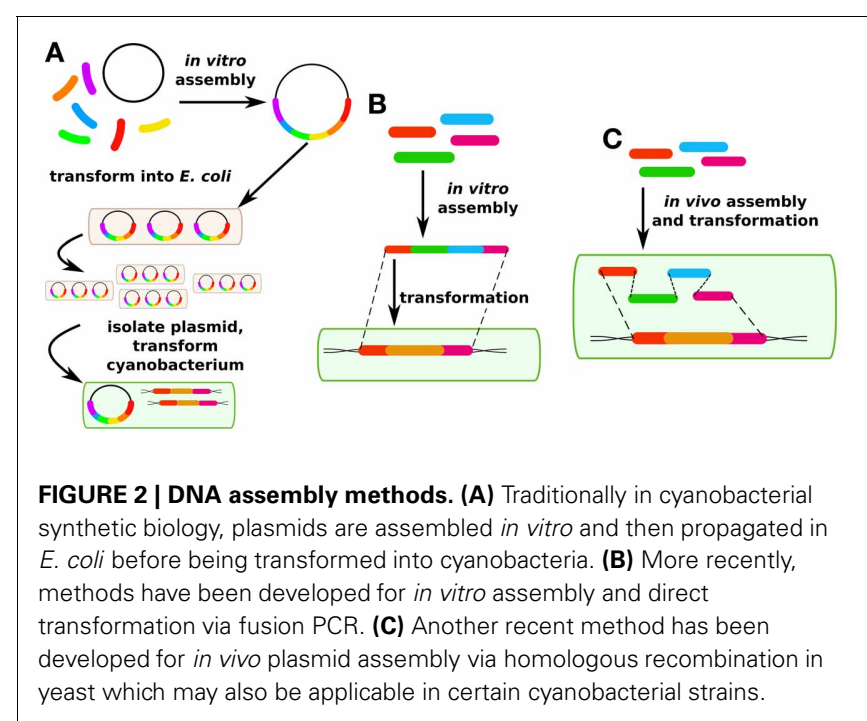


up the process of making cyanobacterial mutant strains, either for modification in cis or in trans. The major technical challenge for such an approach is that the long time after transformation required to isolate cyanobacterial mutants (typically 1 week or more) means it is critical to have high-fidelity assembly methods to avoid a time-consuming screening process.

Although shuttle vectors do exist for cyanobacteria, there has been little characterization of their copy numbers in cyanobacterial hosts, and the lack of replicative vectors with varied copy numbers limits the valuable ability to control the expression level of heterologous genes by selecting their copy number (Jones et al., 2000; Dunlop et al., 2011). Plasmids derived from RSF1010 appear to have a copy number of $10-30$ (or $\sim 1-3$ per chromosome) in Synechocystis sp. PCC 6803 (Ng et al., 2000; Huang et al., 2010), but copy numbers of other broad host-range plasmids have not been quantified to date. Endogenous plasmids of cyanobacteria have also been used as target sites for expression of heterologous genes in Synechococcus sp. PCC 7002 (Xu et al., 2011). This strain harbors several endogenous plasmids whose copy numbers range from $\sim 1$ to 8 per chromosome, with an approximate chromosome copy number of 6 per cell. Synechocystis sp. PCC 6803 also has plasmids whose copy numbers span a similar range [from $\sim 0.4-8$ per chromosome (Berla and Pakrasi, 2012)]. The origins of replication from these plasmids constitute a source of genetic parts that could be used to generate cyanobacterial expression plasmids having a range of copy numbers, and which could potentially be modified to create higher or lower-copy plasmids that are compatible with existing plasmids in various cyanobacterial systems. The range of shuttle vectors that have been used in cyanobacterial hosts has been recently reviewed (Wang et al., 2012). While many tools are available for genetic modification of these biotechnologically promising strains, opportunities abound to develop new and improved tools that will allow research to proceed faster.

\section{UNIOUE CHALLENGES OF THE CYANOBACTERIAL LIFESTYLE}

Organisms that survive using sunlight as a primary nutrient face unique challenges. These must be better understood and addressed to fulfill the biotechnological promise of cyanobacteria through synthetic biology.

\section{LIFE IN A DIURNAL ENVIRONMENT}

A primary goal of synthetic biology in cyanobacteria is to use photosynthesis to convert $\mathrm{CO}_{2}$ into higher-value products such as biofuels and chemical precursors. To make such a process economically and environmentally feasible will require using sunlight as a primary energy source. While some cyanobacteria are facultative heterotrophs, their key advantage over obligate heterotrophic bacteria is photosynthesis. Unlike heterotrophic growth environments where carbon and energy sources can be provided more uniformly both in space and time, sunlight will only be available during the day and will be attenuated as it passes through the culture. Under certain conditions, cultures may be able to take advantage of a "flashing light effect" to integrate spatially uneven illumination by storing chemical energy when in bright light near the reactor surface and using that energy to conduct biochemistry during time spent in the dark away from the reactor surface. This ability will depend on light intensity, mixing rates, reactor geometry, and likely other factors. Certain diazotrophic cyanobacteria can even use daylight to continue growth during the night. Cyanothece sp. ATCC 51142 [along with several other strains (Taniuchi et al., 2008; Latysheva et al., 2012; Pfreundt et al., 2012)] is a unicellular diazotrophic cyanobacterium that performs photosynthesis and accumulates glycogen during the day, and then during the night breaks down its glycogen reserves to supply energy for nitrogen fixation. Thus, these strains spread out the energy available from sunlight over a 24 -h period. This process involves a genome-wide oscillation in transcription, with more than $30 \%$ of genes oscillating in expression between day and night (Stockel et al., 2008). To take full advantage of sunlight, synthetic systems must be created that are capable of responding appropriately to this challenging dynamic environment. It has recently been shown that biofuel-producing strains that dynamically tune the expression of heterologous pathways in response to their own intracellular conditions produce more biofuel and exhibit greater stability of heterologous pathways (Zhang et al., 2012). As challenging as the design of such a system was for batch heterotrophic cultures, it will be even more challenging in production environments that include a diurnal light cycle.

While not all strains exhibit as complete a physiological change between day and night as Cyanothece 51142, all cyanobacteria do have a circadian clock that adapts them to their autotrophic lifestyle. The cyanobacterial circadian clock is anchored by master regulators KaiA, KaiB, and KaiC, which act by cyclically phosphorylating and dephosphorylating each other (Akiyama, 2012). While the circadian rhythm can be reconstituted in vitro using the three Kai proteins in the presence of ATP (Nakajima et al., 2005), the accurate maintenance of this clock in vivo depends on proper protein turnover (Holtman et al., 2005), on codon selection in the kaiBC transcript (Xu et al., 2013), on transcriptional feedback (Teng et al., 2013), and on the controlled response of the entire program of cellular transcription to the output of the KaiABC oscillator. While disturbing rhythmicity can lead to strains that grow better under constant light, the circadian clock is adaptive for strains living in a dynamic environment (Woelfle et al., 2004; Xu et al., 2013). Therefore, integrating synthetic gene circuits such as biofuel production processes into the circadian rhythm of cyanobacterial hosts will likely lead to both improved production and improved strain stability in outdoor production environments.

\section{REDIRECTING CARBON FLUX BY DECOUPLING GROWTH FROM PRODUCTION}

While redirecting carbon flux is a challenge in all metabolic engineering efforts, it has been suggested that stringent control of fixed carbon partitioning among central metabolic pathways poses a major limitation to chemical production especially in photosynthetic organisms (Melis, 2013). During the growth phase, it may be true that carbon partitioning is tightly controlled by any number of mechanisms including metabolite channeling or simply high demand for metabolic intermediates. However, biofuel production during non-growth phases (Atsumi et al., 2009; Liu et al., 2011; Wang et al., 2013) demonstrates that under appropriate conditions, cyanobacterial hosts can produce biofuel 
compounds with higher selectivity, since biofuel can be produced by metabolically active cells even in the absence of growth. Enhancing their productivity in this phase is a major opportunity for cyanobacterial synthetic biologists to overcome these limits on carbon partitioning. Capturing this opportunity will require designing complete metabolic circuits that remain highly active during stationary phase.

\section{RNA-BASED REGULATION}

Recently, regulation of gene expression through RNA mechanisms has received great attention across bacterial clades (Selinger et al., 2000; Sharma et al., 2010; Mitschke et al., 2011). While these mechanisms of regulation may be important in all bacteria, their prominence is perhaps the greatest in the cyanobacteria and may help these diurnal organisms adapt to their highly dynamic environment: in a recent dRNA-seq study, many of the most highly expressed RNAs belonged to families of non-coding RNAs which are present in nearly all sequenced cyanobacteria, but not in any other organisms (Gierga et al., 2009; Mitschke et al., 2011). While their high expression in Synechocystis 6803 suggests functional importance for non-coding RNAs, few have clearly elucidated functions to date. syrl overexpression has been shown to lead to a severe growth defect in Synechocystis 6803 (Mitschke et al., 2011). Another small RNA, $i s i R$, has a critical function in stress response in Synechocystis 6803. isiR binds to the mRNA (isiA) for the iron-stress inducible protein, which when translated, forms a ring around trimers of photosystem I, preventing their activity and thus oxidative stress in the absence of sufficient iron (Duhring et al., 2006). The binding of isiR to isiA appears to result in rapid degradation. This particular arrangement allows a very rapid and emphatic response to iron repletion in cyanobacteria, since a large pool of isiA transcripts can be quickly silenced and marked for degradation by transcription of the antisense $i s i R$. Although little is so far known about the generality of this type of regulation, the dynamics of this response might also be effective to use for synthetic systems in cyanobacteria that live in the presence of light as an intermittently available but critical nutrient.

While non-coding RNA has received a lot of recent attention, two-component systems make up the most widely studied family of environmental response regulators in cyanobacteria. Many of these systems have known functions in response to diverse environmental stimuli such as nitrogen, phosphorous, $\mathrm{CO}_{2}$, temperature, salt, and light intensity and quality (Ashby and Houmard, 2006; Montgomery, 2007). Many of the most widelyused systems in the construction of synthetic biological devices (such as the ara and lux clusters) use 2-component systems, and even combine 2-component systems with non-coding RNA to control system dynamics (Waters and Bassler, 2006). As synthetic biology advances into the construction of more and more complex systems, there will be a growing need to understand and use all of the different mechanisms available for control of gene expression and enzyme activity in cyanobacteria.

\section{PARTS FOR CYANOBACTERIAL SYNTHETIC BIOLOGY}

While cyanobacteria are promising organisms for biotechnology, synthetic biology tools for these organisms lag behind what has been developed for E. coli and yeast (Heidorn et al., 2011). Furthermore, synthetic biology tools developed in E. coli or yeast often do not function as designed in cyanobacteria (Huang et al., 2010). Here, we discuss inducible promoters and reporters in cyanobacteria, and cultivation systems that will allow their testing at increased throughput. Refining such systems will make cyanobacterial synthetic biology more user-friendly, a central goal for developing the "green E. coli."

\section{INDUCIBLE PROMOTERS}

Creation of synthetic biology systems that predictably respond to a specific signal often depends upon inducible promoters for transcriptional control. An ideal inducible promoter will have the following properties: (1) It will not be activated in the absence of inducer. (2) It will produce a predictable response to a given concentration of inducer or repressor. This response may be digital (i.e., on/off) or graded change with different concentrations of inducer/repressor. (3) The inducer at saturating concentrations should have no harmful effect on the host organism. (4) The inducer should be cheap and stable under the growth conditions of the host. Finally, (5) the inducible system should act orthogonally to the host cell's transcriptional program. Ideal transcriptional repressors should not bind to native promoters and if non-native transcriptional machinery is used (such as T7 RNA polymerase) it should not initiate transcription from native promoters. Promoters must perform as ideally as possible in order to be used in the construction of more complex genetic circuits (Moon et al., 2012).

Many common inducible promoters in cyanobacteria respond to transition metals. These have often been the basis of metal detection systems (Erbe et al., 1996; Boyanapalli et al., 2007; Peca et al., 2007, 2008; Blasi et al., 2012). Cyanobacteria balance metal intake for the organisms' needs against potential oxidative stress and protein denaturation (Michel et al., 2001; Peca et al., 2008) via tightly regulated systems. As shown in Table 2, cyanobacteria's metal-responsive promoters frequently show greater than 100-fold dynamic range. For example, the promoter for the Synechocystis sp. PCC 6803 gene, coaA, was induced 500 -fold by $6 \mu \mathrm{M} \mathrm{Co}^{2+}$ (Guerrero et al., 2012), and $P_{\text {smt }}$ from Synechococcus elongatus PCC 7942 was induced 300 -fold by $2 \mu \mathrm{M}$ $\mathrm{Zn}^{2+}$ (Erbe et al., 1996). The most responsive cyanobacterial promoters reported were $\mathrm{P}_{n r s B}$ from Synechocystis sp. PCC 6803, responding 1000 -fold to $0.5 \mu \mathrm{M} \mathrm{Ni}{ }^{2+}$ (Peca et al., 2007), and $\mathrm{P}_{i s i A B}$ also from Synechocystis sp. PCC 6803, repressed 5000-fold by $30 \mu \mathrm{M} \mathrm{Fe}^{3+}$ following depletion (Kunert et al., 2003).

While the sensitivity of these promoters to low concentrations of ions may seem like an advantage, in practice it can make them difficult to use. Glassware must be thoroughly cleaned according to special protocols to remove trace metals and cells often have to be starved for extended periods, inducing stress responses, to use such inducible systems. Additionally, promoters endogenous to a chassis strain are woven into a complex, incompletely understood regulatory system. In this system, promoters are activated by multiple inducers, such as PcoaT $\left(\mathrm{Co}^{2+}\right.$ and $\left.\mathrm{Zn}^{2+}\right)$ and PziaA $\left(\mathrm{Cd}^{2+}\right.$ and $\left.\mathrm{Zn}^{2+}\right)$, both from Synechocystis sp. PCC 6803 and inducers can also activate multiple promoters, such as $\mathrm{Cd}^{2+}$ inducing ziaA and isiA (Blasi et al., 2012). 
Table 2 | Inducible promoters used in cyanobacterial hosts.

\begin{tabular}{|c|c|c|c|c|c|c|c|}
\hline Promoter & Source & $\begin{array}{l}\text { Inducer/repressor } \\
\text { and concentration }\end{array}$ & $\begin{array}{l}\text { Expression } \\
\text { host }\end{array}$ & $\begin{array}{l}\text { Expressed } \\
\text { gene }\end{array}$ & $\begin{array}{l}\text { Dynamic } \\
\text { range }\end{array}$ & $\begin{array}{l}\text { Measure of } \\
\text { expression }\end{array}$ & References \\
\hline \multicolumn{8}{|c|}{ METAL-INDUCIBLE PROMOTERS } \\
\hline ZiaA & $\begin{array}{l}\text { Synechocystis } \\
\text { sp. PCC } 6803\end{array}$ & Inducer $\mathrm{Cd}^{2+} 2 \mu \mathrm{M}$ & $\begin{array}{l}\text { Synechocystis sp. } \\
\text { PCC } 6803\end{array}$ & $z i a A$ & 10 -fold & RT-PCR & Blasi et al., 2012 \\
\hline coat & $\begin{array}{l}\text { Synechocystis } \\
\text { sp. PCC } 6803\end{array}$ & $\begin{array}{l}\text { Inducer } \mathrm{Co}^{2+} \\
6.4 \mu \mathrm{M}\end{array}$ & $\begin{array}{l}\text { Synechocystis sp. } \\
\text { PCC } 6803\end{array}$ & $\operatorname{coa} R+\operatorname{lu} \times A B$ & 70-fold & $70 R L U^{e}$ & Peca et al., 2008 \\
\hline coat & $\begin{array}{l}\text { Synechocystis } \\
\text { sp. PCC } 6803\end{array}$ & Inducer $\mathrm{Co}^{2+} 3 \mu \mathrm{M}$ & $\begin{array}{l}\text { Synechocystis sp. } \\
\text { PCC } 6803\end{array}$ & coaT & 10 -fold & RT-PCR & Peca et al., 2007 \\
\hline petE & $\begin{array}{l}\text { Synechocystis } \\
\text { sp. PCC } 6803\end{array}$ & $\begin{array}{l}\text { Inducer } \mathrm{Cu}^{2+} \\
0.5 \mu \mathrm{M}\end{array}$ & $\begin{array}{l}\text { Synechocystis sp. } \\
\text { PCC } 6803\end{array}$ & $\begin{array}{l}\text { Gene encoding EFE } \\
\text { from Pseudomonas } \\
\text { syringae }\end{array}$ & 5 -fold & $\begin{array}{l}28 \mathrm{~nL} \text { ethylene } \\
\mathrm{mL}^{-1} \mathrm{~h}^{-1}\end{array}$ & $\begin{array}{l}\text { Guerrero et al., } \\
2012\end{array}$ \\
\hline petE & $\begin{array}{l}\text { Synechocystis } \\
\text { sp. PCC } 6803\end{array}$ & Inducer $\mathrm{Cu}^{2+} 3 \mu \mathrm{M}$ & $\begin{array}{l}\text { Anabaena sp. PCC } \\
7120\end{array}$ & hetP & 4.5-fold & $\begin{array}{l}8 \% \text { heterocyst } \\
\text { frequency }\end{array}$ & $\begin{array}{l}\text { Higa and Callahan, } \\
2010\end{array}$ \\
\hline petE & $\begin{array}{l}\text { Synechocystis } \\
\text { sp. PCC } 6803\end{array}$ & $\begin{array}{l}\text { Inducer } \mathrm{Cu}^{2+} \\
0.3 \mu \mathrm{M}\end{array}$ & $\begin{array}{l}\text { Anabaena sp. PCC } \\
7120\end{array}$ & $\begin{array}{l}\text { het } N \text { (prevents } \\
\text { heterocyst formation) }\end{array}$ & $\begin{array}{l}\text { Qualified but } \\
\text { not quantified }\end{array}$ & $\begin{array}{l}0 \% \\
\text { heterocysts } \\
\text { from } 10 \% \\
\text { uninduced }\end{array}$ & $\begin{array}{l}\text { Callahan and } \\
\text { Buikema, } 2001\end{array}$ \\
\hline$i s i A B$ & $\begin{array}{l}\text { Synechocystis } \\
\text { sp. PCC } 6803\end{array}$ & $\begin{array}{l}\text { Repressor } \mathrm{Fe}^{3+} \\
30 \mu \mathrm{M}\end{array}$ & $\begin{array}{l}\text { Synechocystis sp. } \\
\text { PCC } 6803\end{array}$ & $i s i A B+g f p$ & 5000-fold & $\begin{array}{l}\text { From } 5000 \\
\text { RFUe }\end{array}$ & Kunert et al., 2003 \\
\hline$n r s B$ & $\begin{array}{l}\text { Synechocystis } \\
\text { sp. PCC } 6803\end{array}$ & $\begin{array}{l}\text { Inducer } \mathrm{Ni}^{2+} \\
0.5 \mu \mathrm{M}\end{array}$ & $\begin{array}{l}\text { Synechocystis sp. } \\
\text { PCC } 6803\end{array}$ & $n r s B$ & 1000 -fold & RT-PCR & Peca et al., 2007 \\
\hline$n r s B$ & $\begin{array}{l}\text { Synechocystis } \\
\text { sp. PCC } 6803\end{array}$ & Inducer $\mathrm{Ni}^{2+} 5 \mu \mathrm{M}$ & $\begin{array}{l}\text { Synechocystis sp. } \\
\text { PCC } 6803\end{array}$ & $n r s B$ & 400-fold & RT-PCR & Blasi et al., 2012 \\
\hline$n r s B$ & $\begin{array}{l}\text { Synechocystis } \\
\text { sp. PCC } 6803\end{array}$ & $\begin{array}{l}\text { Inducer } \mathrm{Ni}^{2+} \\
6.4 \mu \mathrm{M}\end{array}$ & $\begin{array}{l}\text { Synechocystis sp. } \\
\text { PCC } 6803\end{array}$ & $n r s R+1 u x A B$ & 50-fold & 50 RLUe & Peca et al., 2008 \\
\hline Smt & $\begin{array}{l}\text { Synechococcus } \\
\text { elongatus PCC } \\
7942\end{array}$ & Inducer $\mathrm{Zn}^{2+} 2 \mu \mathrm{M}$ & $\begin{array}{l}\text { Synechococcus } \\
\text { elongatus PCC } \\
7942\end{array}$ & $\begin{array}{l}\text { luxCDABE from Vibrio } \\
\text { fisheri }\end{array}$ & 300 -fold & $\begin{array}{l}325,000 \mathrm{cps} \\
\text { luminescence }\end{array}$ & Erbe et al., 1996 \\
\hline$z i a A$ & $\begin{array}{l}\text { Synechocystis } \\
\text { sp. PCC } 6803\end{array}$ & Inducer $\mathrm{Zn}^{2+} 5 \mu \mathrm{M}$ & $\begin{array}{l}\text { Synechocystis sp. } \\
\text { PCC } 6803\end{array}$ & $z i a A$ & 40-fold & RT-PCR & Peca et al., 2007 \\
\hline$z i a A$ & $\begin{array}{l}\text { Synechocystis } \\
\text { sp. PCC } 6803\end{array}$ & Inducer $\mathrm{Zn}^{2+} 4 \mu \mathrm{M}$ & $\begin{array}{l}\text { Synechocystis sp. } \\
\text { PCC } 6803\end{array}$ & $z i a A$ & 40-fold & RT-PCR & Blasi et al., 2012 \\
\hline coat & $\begin{array}{l}\text { Synechocystis } \\
\text { sp. PCC } 6803\end{array}$ & $\begin{array}{l}\text { Inducer } \mathrm{Zn}^{2+} \\
3.2 \mu \mathrm{M}\end{array}$ & $\begin{array}{l}\text { Synechocystis sp. } \\
\text { PCC } 6803\end{array}$ & $\operatorname{coa} R+\operatorname{lu} x A B$ & 25-fold & 25 RLUe & Peca et al., 2008 \\
\hline coat & $\begin{array}{l}\text { Synechocystis } \\
\text { sp. PCC } 6803\end{array}$ & Inducer $\mathrm{Zn}^{2+} 5 \mu \mathrm{M}$ & $\begin{array}{l}\text { Synechocystis sp. } \\
\text { PCC } 6803\end{array}$ & coaT & 10 -fold & RT-PCR & Peca et al., 2007 \\
\hline
\end{tabular}


Table 2 | Continued

\begin{tabular}{|c|c|c|c|c|c|c|c|}
\hline Promoter & Source & $\begin{array}{l}\text { Inducer/repressor } \\
\text { and concentration }\end{array}$ & $\begin{array}{l}\text { Expression } \\
\text { host }\end{array}$ & $\begin{array}{l}\text { Expressed } \\
\text { gene }\end{array}$ & $\begin{array}{l}\text { Dynamic } \\
\text { range }\end{array}$ & $\begin{array}{l}\text { Measure of } \\
\text { expression }\end{array}$ & References \\
\hline coat & $\begin{array}{l}\text { Synechocystis } \\
\text { sp. PCC } 6803\end{array}$ & Inducer $\mathrm{Zn}^{2+} 4 \mu \mathrm{M}$ & $\begin{array}{l}\text { Synechocystis sp. } \\
\text { PCC } 6803\end{array}$ & coat & 8-fold & RT-PCR & Blasi et al., 2012 \\
\hline Smt & $\begin{array}{l}\text { Synechococcus } \\
\text { elongatus PCC } \\
7002\end{array}$ & Inducer $\mathrm{Zn}^{2+} 2 \mu \mathrm{M}$ & $\begin{array}{l}\text { Synechocystis sp. } \\
\text { PCC } 6803\end{array}$ & $\begin{array}{l}\text { Gene encoding } \\
\text { EFE from } \\
\text { Pseudomonas } \\
\text { syringae }\end{array}$ & 2-fold & $\begin{array}{l}2 \mathrm{~nL} \text { ethylene } \\
\mathrm{mL}^{-1} \mathrm{~h}^{-1}\end{array}$ & $\begin{array}{l}\text { Guerrero et al., } \\
2012\end{array}$ \\
\hline \multicolumn{8}{|c|}{ METABOLITE-INDUCIBLE PROMOTERS } \\
\hline tet $R^{\mathrm{b}}$ & E. coli & $\begin{array}{l}\text { Inducer aTc } \\
10^{3} \mathrm{ng} / \mathrm{per} \mathrm{ml}\end{array}$ & $\begin{array}{l}\text { Synechocystis sp. } \\
\text { strain ATCC27184 }\end{array}$ & eYFP & 290-fold & $>10,000$ RFU & $\begin{array}{l}\text { Huang and } \\
\text { Lindblad, } 2013\end{array}$ \\
\hline $\operatorname{Trc}$ & E. coli & Inducer IPTG $1 \mathrm{mM}$ & $\begin{array}{l}\text { Synechococcus } \\
\text { elongatus PCC } \\
7942\end{array}$ & uidA from E. coli & 36-fold & $\begin{array}{l}340 \mathrm{nmol} \mathrm{MU} \\
\text { min }^{-1} \mathrm{mg} \\
\text { protein }^{-1}(\beta- \\
\text { Glucuronidase } \\
\text { activity) }\end{array}$ & Geerts et al., 1995 \\
\hline A1lacO-1 & E. coli & Inducer IPTG $1 \mathrm{mM}$ & $\begin{array}{l}\text { Synechocystis sp. } \\
\text { PCC } 6803\end{array}$ & $\begin{array}{l}\text { Gene encoding } \\
\text { EFE from } \\
\text { Pseudomonas } \\
\text { syringae }\end{array}$ & 8-fold & $\begin{array}{l}170 \mathrm{~nL} \\
\text { ethylene } \mathrm{mL}^{-1} \\
\mathrm{~h}^{-1}\end{array}$ & $\begin{array}{l}\text { Guerrero et al., } \\
2012\end{array}$ \\
\hline $\operatorname{trc20}$ & E. coli & Inducer IPTG 2 mM & $\begin{array}{l}\text { Synechocystis sp. } \\
\text { PCC } 6803\end{array}$ & $\begin{array}{l}\text { Gene encoding } \\
\text { GFPmut3B }\end{array}$ & 4-fold & $\begin{array}{l}12 \text { RFU } \\
\text { (relative to } \\
\text { Placl) }\end{array}$ & Huang et al., 2010 \\
\hline \multicolumn{8}{|c|}{ MACRONUTRIENT-INDUCIBLE PROMOTERS } \\
\hline psbA2 & $\begin{array}{l}\text { Synechocystis } \\
\text { sp. PCC6803 }\end{array}$ & $\begin{array}{l}\text { Inducer light } \\
500 \mu \mathrm{mol} \text { photons } \\
\mathrm{m}^{-2} \mathrm{~s}^{-1}\end{array}$ & $\begin{array}{l}\text { Synechocystis sp. } \\
\text { PCC6803 }\end{array}$ & $\begin{array}{l}\text { ispS from Pueraria } \\
\text { montana (kudzu) }\end{array}$ & $\begin{array}{l}\text { Qualified but } \\
\text { not quantified }\end{array}$ & $\begin{array}{l}\sim 50 \mathrm{mg} \\
\text { isoprene } \mathrm{g} \\
\mathrm{DCW}^{-1} \mathrm{~d}^{-1}\end{array}$ & $\begin{array}{l}\text { Lindberg et al., } \\
2010\end{array}$ \\
\hline$p s b A 2^{a}$ & $\begin{array}{l}\text { Synechocystis } \\
\text { sp. PCC } 6803\end{array}$ & $\begin{array}{l}\text { Inducer light } \\
50 \mu \mathrm{Em}^{-2} \mathrm{~s}^{-1}\end{array}$ & $\begin{array}{l}\text { Synechocystis sp. } \\
\text { PCC } 6803\end{array}$ & $\begin{array}{l}\text { hydA1 from } \\
\text { Chlamydomonas } \\
\text { reinhardtii }\end{array}$ & $\begin{array}{l}\text { Qualified but } \\
\text { not quantified }\end{array}$ & $\begin{array}{l}130 \mathrm{nmol}^{-1} \\
\mathrm{H}_{2} \mathrm{mg} \mathrm{Chl}^{-1} \\
\min ^{-1}\end{array}$ & Berto et al., 2011 \\
\hline$p s b A 1$ & $\begin{array}{l}\text { Anabaena sp. } \\
\text { PCC } 7120\end{array}$ & $\begin{array}{l}\text { Inducer light } \\
30 \mu \mathrm{Em}^{-2} \mathrm{~s}^{-1}\end{array}$ & $\begin{array}{l}\text { Anabaena sp. PCC } \\
7120\end{array}$ & het $R$ from E. coli & & $\begin{array}{l}17 \% \\
\text { heterocyst } \\
\text { frequency }\end{array}$ & $\begin{array}{l}\text { Chaurasia and } \\
\text { Apte, } 2011\end{array}$ \\
\hline nirA & $\begin{array}{l}\text { Synechococcus } \\
\text { elongatus PCC } \\
7942\end{array}$ & $\begin{array}{l}\text { Inducer/Repressor } \\
\mathrm{NO}_{3}^{-} / \mathrm{NH}_{4}^{+} \\
17.6 \mathrm{mM} / 17.6 \mathrm{mM}\end{array}$ & $\begin{array}{l}\text { Synechocystis sp. } \\
\text { PCC } 6803\end{array}$ & $\begin{array}{l}\text { Gene encoding } \\
\text { p-hydroxyphenylpy } \\
\text { ruvate dioxygenase } \\
\text { from Arabidopsis } \\
\text { thaliana }\end{array}$ & 25-fold & $\begin{array}{l}250 \mathrm{ng} \\
\text { tocopherol mg } \\
\mathrm{DCW}^{-1}\end{array}$ & Qi et al., 2005 \\
\hline
\end{tabular}


Table 2 | Continued

\begin{tabular}{|c|c|c|c|c|c|c|c|}
\hline Promoter & Source & $\begin{array}{l}\text { Inducer/repressor } \\
\text { and concentration }\end{array}$ & $\begin{array}{l}\text { Expression } \\
\text { host }\end{array}$ & $\begin{array}{l}\text { Expressed } \\
\text { gene }\end{array}$ & $\begin{array}{l}\text { Dynamic } \\
\text { range }\end{array}$ & $\begin{array}{l}\text { Measure of } \\
\text { expression }\end{array}$ & References \\
\hline nirA & $\begin{array}{l}\text { Synechococcus } \\
\text { elongatus PCC } \\
7942\end{array}$ & $\begin{array}{l}\text { Inducer/Repressor } \\
\mathrm{NO}_{3}^{-} / \mathrm{NH}_{4}^{+} \\
15.0 \mathrm{mM} / 3.75 \mathrm{mM}\end{array}$ & $\begin{array}{l}\text { Synechococcus } \\
\text { elongatus PCC } \\
7942\end{array}$ & cmpABCD & 5-fold & $\begin{array}{l}260 \mathrm{nmol} \\
\mathrm{HCO}_{3}^{-} \mathrm{mg} \\
\mathrm{Chl}^{-1}\end{array}$ & Omata et al., 1999 \\
\hline Nir & $\begin{array}{l}\text { Anabaena sp. } \\
\text { PCC } 7120\end{array}$ & $\begin{array}{l}\text { Inducer/Repressor } \\
\mathrm{NO}_{3}^{-} / \mathrm{NH}_{4}^{+} \\
5.9 \mathrm{mM} / 10.0 \mathrm{mM}\end{array}$ & $\begin{array}{l}\text { Anabaena sp. PCC } \\
7120\end{array}$ & nir & $\begin{array}{l}\text { Qualified but } \\
\text { not quantified }\end{array}$ & $\begin{array}{l}250 \mathrm{mg} \text { labeled } \\
\text { proteins for } \\
\mathrm{NMR} \mathrm{L}^{-1}\end{array}$ & $\begin{array}{l}\text { Desplancq et al., } \\
2005\end{array}$ \\
\hline
\end{tabular}

aln the presence of $5 \mu M D C M U$, which inhibits the PSII-dependent oxygen evolution.

${ }^{b}$ Grown in the dark on $5 \mathrm{mM}$ glucose.

c Leaky production of 2,3-butanediol, no IPTG, and 1 mM IPTG similar.

dPlac variants had differential expression early in growth phase but dynamic range was reduced as growth proceeded.

${ }^{e} R F U=$ Relative Fluorescence Units; $R L U=$ Relative Luminescence Units.

Thus, these promoters fall short according to criteria 2, 3, and 5 described above.

While few good choices have so far been available for inducible promoters in cyanobacteria, it will be helpful to understand the differences in the cellular machinery of E. coli and cyanobacteria in order to adapt existing systems for use in a cyanobacterial "green E. coli." First, RNA polymerase (RNAP) is structurally different between $E$. coli and cyanobacteria. In cyanobacteria the $\beta$ ' subunit of the RNAP holoenzyme is split into two parts, as opposed to one in most eubacteria, creating a different DNA binding domain (Imamura and Asayama, 2009). Being photosynthetic, circadian, and sometimes nitrogen-fixing, cyanobacteria also employ three sets of interconnected $\sigma$ factors that are different than those used by E. coli (Imamura and Asayama, 2009). Guerrero et al. (2012) looked at the variation in the -35 and -10 regions of $\mathrm{P}_{\mathrm{AllacO}-1}$ and $\mathrm{P}_{\text {trc }}$. $\mathrm{P}_{\text {trc }}$ is not inducible in Synechocystis sp PCC 6803 and had the "standard" bacterial structure in these regions while $\mathrm{P}_{\mathrm{AllacO}-1}$, which produced an 8-fold response to IPTG in the same host, had a different structure in both regions. They postulated that Synechocystis 6803's sigma factors had different selectivity for these two regions. In fact, by systematically altering the bases between -10 and the transcription start site, a library of TetR-regulated promoters with improved inducibility were created in Synechocystis sp. strain ATCC27184 (a glucose-tolerant derivative of Synechocystis 6803). The best performing promoter induced a 290-fold change in response to $1 \mu \mathrm{g} / \mathrm{ml}$ aTc (Huang and Lindblad, 2013). This work demonstrates the improvements that can be seen when modifying parts to work in a particular chassis. However, the light-sensitivity of the inducer aTc required the use of special growth lights that may have had other effects on photoautotrophic metabolism. Further studies that follow in this vein of using well-characterized synthetic biology parts and modifying them to function optimally in a particular cyanobacterial chassis are likely to bear fruit.

The lack of inducibility seen in lac-derived promoters in cyanobacteria could also be a function of inadequate transport of IPTG into cells. Concentrations of IPTG above $1 \mathrm{mM}$ have been shown to induce lac-derived promoters in organisms without an active lactose permease, like many cyanobacteria.
By introducing an active lactose permease into Pseudomonas fluorescens, inducibility was boosted five times at $0.1 \mathrm{mM}$ IPTG (Hansen et al., 1998). Evolving the Lac repressor for improved inducibility is another strategy. Gene expression improved ten times with $1 \mu \mathrm{M}$ IPTG through rounds of error prone PCR and DNA shuffling (Satya Lakshmi and Rao, 2009). Strength of expression and inducibility may also vary between different cyanobacterial strains. IPTG caused as much as a 36-fold response using the trc promoter in Synechococcus elongatus PCC 7942, but little or no response in Synechocystis sp. PCC 6803 (See Table 2). Phylogenetic analysis of $\sigma$ factors from six different cyanobacterial strains, including Synechocystis 6803, showed S. elongatus 7942 to be distinctive. S. elongatus 7942 has $\sigma$ factors that are unique to marine cyanobacteria as well as a group $3 \sigma$ factor similar to those from the heterocyst-forming Anabaena sp. PCC 7120 (Imamura and Asayama, 2009). Understanding these strainspecific differences will enhance the synthetic biologist's ability to design promoters with ideal characteristics in their chassis of choice. This relates to the ability to take up inducers as well as the optimal characteristics of inducers (as in the light-sensitivity of aTc) as described above.

\section{REPORTERS}

Characterization of synthetic biological circuits depends on a reporting method to track the expression, interaction, and position of proteins. Preferably the reporter should be detected without destruction of the organisms or additional inputs. Bacterial luciferase and fluorescent proteins are the most common noninvasive reporters. The lux operon is frequently used for reporting in cyanobacteria (Michel et al., 2001; Mackey et al., 2007; Peca et al., 2008) and is well-suited for real time reporting of gene expression due to the short half-life of the relevant enzymes (Ghim et al., 2010). The superior brightness of fluorescent proteins makes them more ideal for subcellular localization via microscopy or for cell-sorting methods. Fluorescent proteins are produced in an array of colors and also do not require additional substrates. Their use in cyanobacteria is somewhat complicated by the fluorescence of the organism's photosynthetic pigments, but Cerulean, GFPmut3B (a mutant of green fluorescent protein) and EYFP (enhanced yellow fluorescent protein) have all been 
used successfully in cyanobacteria as reporters of gene expression (Huang et al., 2010; Heidorn et al., 2011; Landry et al., 2012; Huang and Lindblad, 2013).

Bacterial luciferase luminesces upon oxidation of reduced flavin mononucleotide (Meighen, 1993). Fluorescent proteins also require oxygen to correctly-fold and fluoresce (Hansen et al., 2001). The light-dark cycle of nitrogen-fixing cyanobacteria provides temporal separation of the oxygen-sensitive nitrogenase from oxygen-evolving photosynthesis (Golden et al., 1997). During the dark cycle, respiration reduces intra-cellular oxygen levels so that nitrogenase can function. Therefore, neither bacterial luciferase nor traditional fluorescent proteins can likely be used to study cyanobacteria in their dark cycle or to report on synthetic biology systems that operate in these oxygen-depleted conditions. Using blue light photoreceptors from Bacillus subtilis and Pseudomonas putida, oxygenindependent flavin mononucleotide-binding florescent proteins have been devised (Drepper et al., 2007). With an excitation wavelength of $450 \mathrm{~nm}$ and an emission wavelength of $495 \mathrm{~nm}$, they should perform well in cyanobacteria, although no data supporting this has been published yet. Functionality of these new fluorescent proteins was also improved by replacing a phenylalanine suspected of quenching with serine or threonine, resulting in a doubling of the brightness (Mukherjee et al., 2012). This expanding variety of easily readable reporter systems will be extremely valuable for cyanobacterial synthetic biology.

\section{CULTIVATION SYSTEMS}

To date, most synthetic biology and metabolic engineering work in cyanobacteria has been performed using simple, low-tech cultivation methods such as shake flasks or bubbling tubes grown under standard fluorescent light sources. Often, laboratory incubators have simply been retrofitted by the addition of fluorescent light sources available in home improvement stores. However, as light and $\mathrm{CO}_{2}$ are major nutrients for cyanobacteria, it is critical to properly standardize the inputs of these resources to reliably characterize biological parts. It is also critical to increase the throughput of cyanobacterial growth systems to be able to screen the large numbers of variants that can be generated by combinatorial methods, as is routinely performed by growing heterotrophic bacterial cultures in 96-well plate format. Growth of cyanobacteria in 6-well plates can be routinely performed in our lab and by others (Huang and Lindblad, 2013) along with 24-well plates (Simkovsky et al., 2012), but growth in 96-well plates is poor, limiting assay throughput and requiring more space in lighted chambers under consistent illumination, which is often a limitation. Simple, low-cost systems to reproducibly grow many cyanobacterial cultures in parallel are necessary.

\section{GENOME-SCALE MODELING AND FLUXOMICS OF CYANOBACTERIA}

A primary aim of cyanobacterial synthetic biology is the production of particular metabolites as biofuels or platform chemicals. As such, better understanding the metabolic phenotypes of wildtype and synthetic strains is a critical aim. While cyanobacterial metabolomics have been recently reviewed (Schwarz et al., 2013),

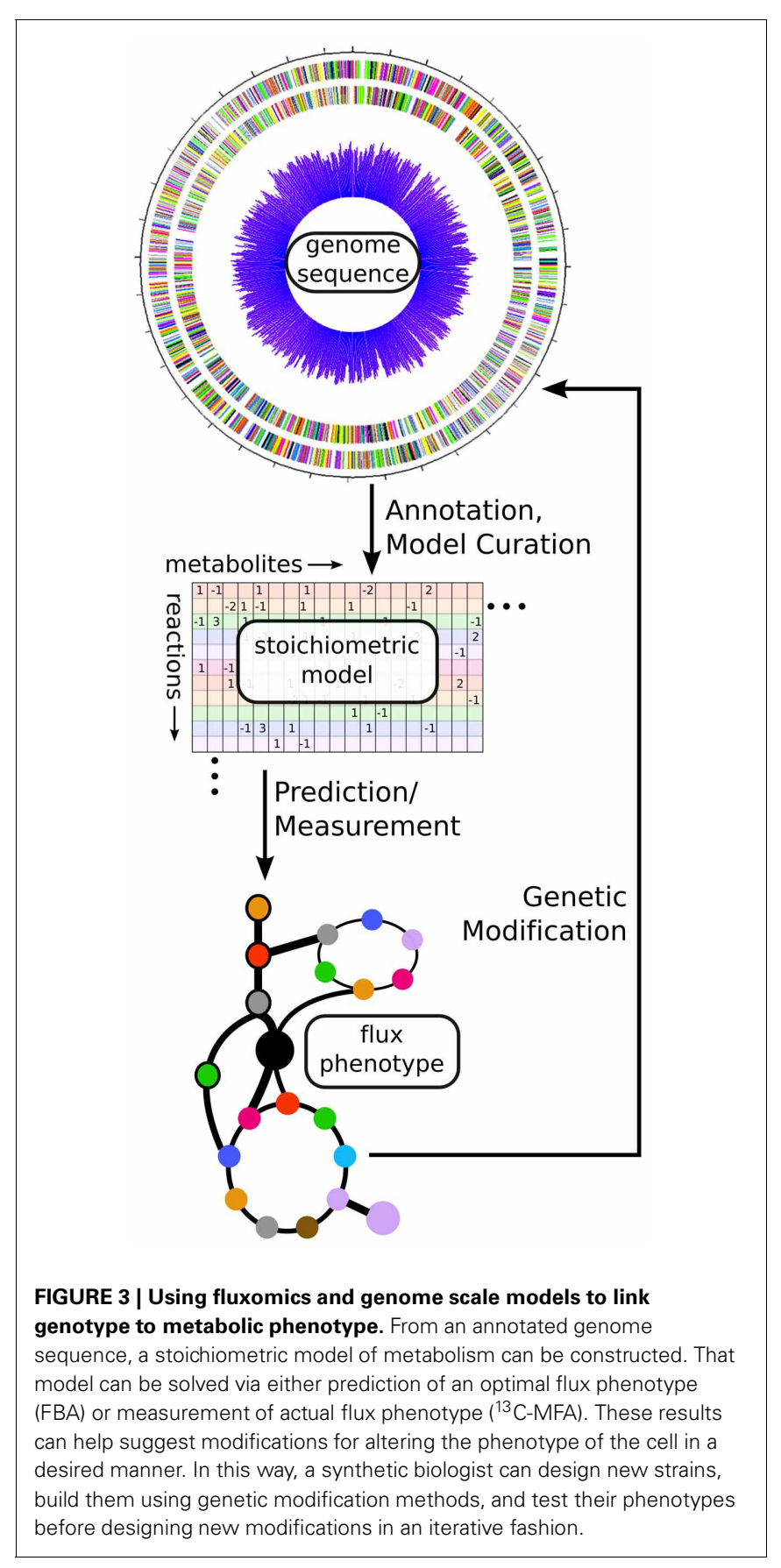

here we describe recent progress in genome-scale modeling and fluxomics of cyanobacteria. These approaches can help guide the creation of synthetic strains with desirable metabolic phenotypes such as biofuel overproduction via in silico prediction or in vivo measurement of metabolic fluxes (See Figure 3). Specific to cyanobacterial systems, we highlight a number of challenges including complexity of modeling the photosynthetic metabolism and performing flux balance analysis (FBA), poor annotations of important metabolic pathways, and unavailability of in vivo gene essentiality information for most cyanobacteria. Finally, we focus on recent advancements in this area. 


\section{CHALLENGES}

\section{Incorporating photoautotrophy into metabolic models}

FBA is a tool to make quantitative in silico predictions about metabolism (Fell and Small, 1986; Savinell and Palsson, 1992; Varma et al., 1993; Orth et al., 2010). An FBA model incorporates the stoichiometry of all genome-encoded metabolic reactions and assumes steady-state growth, such as during exponential phase. This assumption leads to a model that consists of a system of algebraic equations which state that the rate of producing any given metabolite is equal to the rate of consuming that metabolite. A solution to this system of equations is a possible answer to the question "what are all the metabolic fluxes in this system?" Since there are usually more reactions than metabolites, this system of equations is underdetermined and has many possible solutions. Therefore, one has to pick a solution that satisfies a biological objective, such as maximal growth, energy production, or byproduct formation (Varma and Palsson, 1994). For this purpose, a model will also include upper and lower bounds of fluxes that constrain the model to produce physically and biologically reasonable solutions.

Success of FBA greatly depends on the quality of the metabolic network reconstruction as well as the availability of regulatory constraints under a given environmental or growth condition. For instance, constraints can be added that disable or limit fluxes due to known regulatory constraints or substrate availability (Zomorrodi et al., 2012). For cyanobacteria, the major challenges to develop a genome-scale metabolic model and subsequently perform FBA are the same ones faced by these organisms in their diurnal environment: how to incorporate light and how to differentiate light and dark metabolisms. Although it has been nearly a decade since publication of the first study applying FBA to cyanobacteria, it is only recently that models have incorporated complete descriptions of the light reactions of photosynthesis (Nogales et al., 2012). In so doing, these authors were able to highlight the critical importance of alternate electron flow pathways to growth under diverse environmental conditions, and to identify differences in metabolism during carbon-limited and light-limited growth. However, debate remains among photosynthesis researchers about the exact form of the light reactions (Heyes and Hunter, 2005; Kopecna et al., 2013). This uncertainty about the exact stoichiometry of metabolism is a challenge for the predictive power of FBA in photosynthetic systems. While FBA requires the assumption of a pseudo-steady state, all cyanobacteria must alternate between day and night metabolisms during a diurnal cycle. A recent model (Saha et al., 2012) of Cyanothece sp. ATCC 51142 utilizes proteomic data to model the diurnal rhythm of this strain, which fixes carbon during the day and nitrogen during the night (see section Unique Challenges of the Cyanobacterial Lifestyle).

\section{Incompleteness of genome annotation}

Genome scale models are built starting with an annotated genome sequence (see Figure 3), which allows prediction of which metabolic reactions are available in a given strain. However, genome annotation is constantly evolving, and open questions remain about important metabolic reactions in cyanobacteria.
The understanding of several key pathways in cyanobacteria has been recently revised. Zhang and Bryant (2011) identified enzymes from Synechococcus 7002 that can complete the TCA cycle in vitro and have homologues in most cyanobacterial species, which were previously thought to possess an incomplete TCA cycle. Based on this information, Synechocystis 6803 model iSyn731 (Saha et al., 2012) allows for a complete TCA cycle including these reactions. However, using flux variability analysis (Mahadevan et al., 2002; Mahadevan and Schilling, 2003) it was determined that this alternate pathway is not essential for maximal biomass production [unpublished results, (Saha et al., 2012)]. Fatty acid metabolism in cyanobacteria has unique properties that have been recently uncovered due to increased interest in these pathways for biofuel production. Both Synechocystis sp. PCC 6803 and Synechococcus elongatus PCC 7942 contain a single candidate gene annotated for fatty acid activation. While in both organisms the gene is annotated as acyl-CoA synthetase, it shows only acyl-ACP synthetase activity instead (Kaczmarzyk and Fulda, 2010). Further analysis also shows the importance of acyl-ACP synthetase in enabling the transfer of fatty acids across the membrane (Von Berlepsch et al., 2012). Quinone synthesis is another pathway with conflicting annotations. Cyanobacteria contain neither ubiquinone nor menaquinone (Collins and Jones, 1981). Despite the lack of ubiquinone within cyanobacteria, a number of cyanobacterial genomes contain homologs for six $E$. coli genes involved in ubiquinone biosynthesis (Sakuragi, 2004). Given these homologous genes it is probable that plastoquinone, a quinone molecule participating in the electron transport chain, is produced in cyanobacteria using a pathway very similar to that of ubiquinone production in proteobacteria. Wu et al. (2010) showed that Cyanothece 51142 contains an alternative pathway for isoleucine biosynthesis. Threonine ammonia-lyase, catalyzing the conversion of threonine to 2-ketobutyrate, is absent in Cyanothece 51142. Instead, this organism uses a citramalate pathway with pyruvate and acetyl-CoA as precursors for isoleucine synthesis. An intermediate in this pathway, namely ketobutyrate, can be converted to higher alcohols (propanol and butanol) via this nonfermentative alcohol production pathway. These active areas of research will help to better define cyanobacterial metabolism and allow the generation of models that can more accurately predict cellular phenotypes. While newer fluxomics techniques can yield powerful results in well-characterized strains, developing a "green E. coli" will also require expanded knowledge of biochemistry that to date can only come from older methods of single gene or single protein analysis.

\section{Fewer mutant resources to test model accuracy}

The quality or accuracy of any genome-scale metabolic model can be tested by contrasting the in silico growth phenotype with available experimental data on the viability of single or multiple gene knockouts (Thiele and Palsson, 2010). Any discrepancies between model predictions and observed results can aid in model refinement (Kumar and Maranas, 2009). For model strains besies cyanobacteria, concerted efforts to create complete mutant libraries have led to improvements in metabolic modeling. To the best of our knowledge, extensive in vivo gene essentiality data are available only for Synechocystis 6803 among the cyanobacteria in 
the CyanoMutants database (Nakamura et al., 1999; Nakao et al., 2010), but only for $\sim 119$ genes, compared with 731 genes associated with metabolic reactions in a recent genome-scale model (Saha et al., 2012). Thus, only a small subset of the model predictions on gene essentiality can be evaluated using available data for Synechocystis 6803, and the proportion is much less for any other strain. While a genome-wide library of knockout mutants has been created in Synechococcus 7942 (Chen et al., 2012) segregation (and thus essentiality) has only been checked for a small selection of these mutants and its not available in any large-scale public database to date. Unavailability of such mutant information limits model validation and in turn hurts the value of computational predictions from FBA. Efforts to create complete mutant libraries in model cyanobacterial strains would improve the fidelity of genome-scale metabolic models, leading to testable hypotheses about how to alter metabolism for metabolite overproduction.

\section{RECENT ADVANCES}

\section{Detailed genome-scale models}

Genome-scale models contain detailed Gene-Protein-Reaction associations, a stoichiometric representation of all possible reactions occurring in an organism, and a set of appropriate regulatory constraints on each reaction flux. They are differentiated from more basic FBA models simply by their completenessthey span all or nearly all of the metabolic reactions encoded in a genome. Thus, these models can have greater predictive value than those of only central metabolism. Among cyanobacteria, Cyanothece 51142 exhibits one of the highest rates of nitrogen fixation and is the first unicellular diazotrophic cyanobacterium to be completely sequenced (Welsh et al., 2008). The first genomescale model for Cyanothece 51142, iCce806, is recently developed (Vu et al., 2012), while another more recent genome-scale model $i$ Cyt773 contains an additional 266 unique reactions spanning pathways such as lipid, pigment and alkane biosynthesis (Saha et al., 2012). iCyt773 also models diurnal metabolism by including flux regulation based on available day/night protein expression data (Stockel et al., 2011) and developing separate (light/dark) biomass equations. These models greatly enhance the ability to make computational predictions about this unique and promising diazotrophic organism.

Since Synechocystis 6803 is a model cyanobacterial strain, it has long been the target for modeling of photosynthetic central metabolism (Yang et al., 2002; Shastri and Morgan, 2005). More recent models (Knoop et al., 2010; Montagud et al., 2011) analyze growth under different conditions and detect bottlenecks and gene knock-out candidates to enhance metabolite production (e.g., ethanol, succinate, and hydrogen). A recent model represents the photosynthetic apparatus in detail, detects alternate flow pathways of electrons and also pinpoints photosynthetic robustness during photoautotrophic metabolism (Nogales et al., 2012). iSyn731, the latest of all Synechocystis 6803 models, integrates all recent developments and supplements them with improved metabolic capability and additional literature evidence. As many as 322 unique reactions are introduced in iSyn731 including reactions distributed in pathways such as heptadecane and fatty acid biosynthesis (Saha et al., 2012). Furthermore, iSyn731 is the first model for which both gene essentiality data (Nakamura et al., 1999) and MFA flux data (Young et al., 2011) are utilized to assess the predictive quality. Additionally, genome scale modeling has been extended to include another model cyanobacterium, Synechococcus sp. PCC 7002 (Hamilton and Reed, 2012). Other model strains highlighted in Table 1 have not yet had genome-scale models generated for their metabolism. Thus, stoichiometric models are emerging as a valuable tool for use across model cyanobacterial systems.

\section{${ }^{13}$ C MFA analysis}

While in silico models are great tools for generating hypotheses on how to use synthetic biology interventions to alter metabolism, they need to be complemented by fluxomics methods that allow in vivo measurement of metabolic fluxes to assess these interventions. Such a suite of tools allows the closure of the designbuild-test engineering cycle in synthetic biology (Figure 3). To this end, Young et al. (2011) have developed a method to measure fluxes in autotrophic metabolism via dynamic isotope labeling measurements. In this approach, cultures are fed with a stepchange from naturally labeled bicarbonate to $\mathrm{NaH}^{13} \mathrm{CO}_{3}$ and the labeling patterns of metabolic intermediates are followed over a time-course to determine relative rates of metabolic flux. Previous studies (Yang et al., 2002) have also assessed metabolic fluxes under mixotrophic growth conditions, using a pseudosteady-state approach in which cells are fed with ${ }^{13} \mathrm{C}$ labeled glucose and metabolic fluxes are inferred from labeling patterns of proteinogenic amino acids. These studies have been extremely useful in identifying fluxes that exist in vivo, but have previously been regarded as wasteful or futile cycles, such as the oxidative pentose phosphate pathway and RuBP oxygenation. Comparisons between flux measurements (Young et al., 2011) and flux predictions (Saha et al., 2012) for Synechocystis 6803 have revealed the necessity of additional regulatory information for accurate in silico predictions of phenotype. These modeling and fluxomics efforts have resulted in deeper understanding of the metabolic capabilities of the modeled strains and of cyanobacteria in general.

\section{CONCLUSIONS}

Cyanobacterial synthetic biology offers great promise for enhancing efforts to produce biofuels and chemicals in photoautotrophic hosts. While several cyanobacterial chassis strains have been used in synthetic biology efforts, the tools for their manipulation and analysis need greater development to unlock this potential and develop a "green E. coli." Metabolic modeling is a complementary tool that can help guide the creation of synthetic strains with desirable phenotypes. By developing the tools for strain manipulation and control, synthetic biologists can unlock a bright future for the biotechnological use of abundant light and $\mathrm{CO}_{2}$.

\section{ACKNOWLEDGMENTS}

We thank members of the Pakrasi, Maranas, and Moon labs for collegial discussions. Development of this manuscript was supported by funding from the Office of Science (BER), U. S. Department of Energy to Drs. Himadri B. Pakrasi and Costas D. Maranas, and a National Science Foundation Graduate Research Fellowship to Cheryl M. Immethun. 


\section{REFERENCES}

Akiyama, S. (2012). Structural and dynamic aspects of protein clocks: how can they be so slow and stable? Cell. Mol. Life Sci. 69, 2147-2160. doi: 10.1007/s00018-012-0919-3

Ashby, M. K., and Houmard, J. (2006). Cyanobacterial two-component proteins: structure, diversity, distribution, and evolution. Microbiol. Mol. Biol. Rev. 70, 472-509. doi: 10.1128/MMBR.00046-05

Atsumi, S., Higashide, W., and Liao, J. C. (2009). Direct photosynthetic recycling of carbon dioxide to isobutyraldehyde. Nat. Biotechnol. 27, 1177-1180. doi: 10.1038/nbt.1586

Berla, B. M., and Pakrasi, H. B. (2012). Upregulation of plasmid genes during stationary phase in Synechocystis sp. strain PCC 6803, a cyanobacterium. Appl. Environ. Microbiol. 78, 5448-5451. doi: 10.1128/AEM.01174-12

Berto, P., D'adamo, S., Bergantino, E., Vallese, F., Giacometti, G. M., and Costantini, P. (2011). The cyanobacterium Synechocystis sp. PCC 6803 is able to express an active $[\mathrm{FeFe}]$-hydrogenase without additional maturation proteins. Biochem. Biophys. Res. Commun. 405, 678-683. doi: 10.1016/j.bbrc.2011.01.095

Blasi, B., Peca, L., Vass, I., and Kos, P. B. (2012). Characterization of stress responses of heavy metal and metalloid inducible promoters in Synechocystis PCC6803. J. Microbiol. Biotechnol. 22, 166-169. doi: 10.4014/jmb.1106.06050

Boyanapalli, R., Bullerjahn, G. S., Pohl, C., Croot, P. L., Boyd, P. W., and McKay, R. M. (2007). Luminescent whole-cell cyanobacterial bioreporter for measuring $\mathrm{Fe}$ availability in diverse marine environments. Appl. Environ. Microbiol. 73, 1019-1024. doi: 10.1128/AEM.01670-06

Callahan, S. M., and Buikema, W. J. (2001). The role of HetN in maintenance of the heterocyst pattern in Anabaena sp. PCC 7120. Mol. Microbiol. 40, 941-950. doi: 10.1046/j.1365-2958.2001.02437.x

Chaurasia, A. K., and Apte, S. K. (2011). Improved eco-friendly recombinant Anabaena sp. strain PCC7120 with enhanced nitrogen biofertilizer potential. Appl. Environ. Microbiol. 77, 395-399. doi: 10.1128/AEM.01714-10

Cheah, Y. E., Albers, S. C., and Peebles, C. A. (2013). A novel counter-selection method for markerless genetic modification in Synechocystis sp. PCC 6803.
Biotechnol. Prog. 29, 23-30. doi: 10.1002/btpr.1661

Chen, Y., Holtman, C. K., Taton, A., and Golden, S. S. (2012). "Functional analysis of the synechococcus elongatus PCC 7942 Genome," in Functional Genomics and Evolution of Photosynthetic Systems, eds R. Burnap and W. Vermaas (Springer), 119-137. Available online at: http:// www.springer.com/life+sciences/bo ok/978-94-007-1532-5

Collins, M. D., and Jones, D. (1981). Distribution of isoprenoid quinone structural types in bacteria and their taxonomic implications. Microbiol. Rev. 45, 316-354.

Desplancq, D., Bernard, C., Sibler, A. P., Kieffer, B., Miguet, L., Potier, N., et al. (2005). Combining inducible protein overexpression with NMRgrade triple isotope labeling in the cyanobacterium Anabaena sp. PCC 7120. Biotechniques 39, 405-411. doi: 10.2144/05393RR02

Drepper, T., Eggert, T., Circolone, F., Heck, A., Krauss, U., Guterl, J. K., et al. (2007). Reporter proteins for in vivo fluorescence without oxygen. Nat. Biotechnol. 25, 443-445. doi: $10.1038 /$ nbt1293

Duhring, U., Axmann, I. M., Hess, W. R., and Wilde, A. (2006). An internal antisense RNA regulates expression of the photosynthesis gene isiA. Proc. Natl. Acad. Sci. U.S.A. 103, 7054-7058. doi: 10.1073/pnas.0600927103

Dunlop, M. J., Dossani, Z. Y., Szmidt, H. L., Chu, H. C., Lee, T. S., Keasling, J. D., et al. (2011). Engineering microbial biofuel tolerance and export using efflux pumps. Mol. Syst. Biol. 7, 487. doi: $10.1038 / \mathrm{msb} .2011 .21$

Engler, C., and Marillonnet, S. (2011). Generation of families of construct variants using golden gate shuffling. Methods Mol. Biol. 729, 167-181. doi: 10.1007/978-1-61779-065-2_11

Erbe, J. L., Adams, A. C., Taylor, K. B., and Hall, L. M. (1996). Cyanobacteria carrying an smt-lux transcriptional fusion as biosensors for the detection of heavy metal cations. J. Ind. Microbiol. 17, 80-83. doi: 10.1007/BF01570047

Fell, D. A., and Small, J. R. (1986). Fat synthesis in adipose tissue. An examination of stoichiometric constraints. Biochem. J. 238, 781-786.

Geerts, D., Bovy, A., De Vrieze, G., Borrias, M., and Weisbeek, P. (1995). Inducible expression of heterologous genes targeted to a chromosomal platform in the cyanobacterium Synechococcus sp. PCC 7942.
Microbiology 141(Pt 4), 831-841. doi: 10.1099/13500872-141-4-831

Ghim, C. M., Lee, S. K., Takayama, S., and Mitchell, R. J. (2010). The art of reporter proteins in science: past, present and future applications. BMB Rep. 43, 451-460. doi: 10.5483/BMBRep.2010.43.7.451

Gibson, D. G., Young, L., Chuang, R. Y., Venter, J. C., Hutchison, C. A. 3rd., and Smith, H. O. (2009). Enzymatic assembly of DNA molecules up to several hundred kilobases. Nat. Methods 6, 343-345. doi: 10.1038/nmeth. 1318

Gierga, G., Voss, B., and Hess, W. R. (2009). The Yfr2 ncRNA family, a group of abundant RNA molecules widely conserved in cyanobacteria. RNA Biol. 6, 222-227. doi: 10.4161/rna.6.3.8921

Golden, S. S., Ishiura, M., Johnson, C. H., and Kondo, T. (1997). Cyanobacterial Circadian Rhythms. Annu. Rev. Plant Physiol. Plant Mol. Biol. 48, 327-354. doi: 10.1146/annurev.arplant.48.1.327

Gronenberg, L. S., Marcheschi, R. J., and Liao, J. C. (2013). Next generation biofuel engineering in prokaryotes. Curr. Opin. Chem. Biol. doi: 10.1016/j.cbpa.2013.03.037

Guerrero, F., Carbonell, V., Cossu, M., Correddu, D., and Jones, P. R (2012). Ethylene synthesis and regulated expression of recombinant protein in Synechocystis sp. PCC 6803. PLoS ONE 7:e50470. doi: 10.1371/journal.pone.0050470

Hamilton, J. J., and Reed, J. L. (2012). Identification of functional differences in metabolic networks using comparative genomics and constraint-based models. PLoS ONE 7:e34670. doi: 10.1371/journal.pone.0034670

Hansen, L. H., Knudsen, S., and Sorensen, S. J. (1998). The effect of the lacY gene on the induction of IPTG inducible promoters, studied in Escherichia coli and Pseudomonas fluorescens. Curr. Microbiol. 36, 341-347. doi: $10.1007 / \mathrm{s} 002849900320$

Hansen, M. C., Palmer, R. J. Jr., Udsen, C., White, D. C., and Molin, S. (2001). Assessment of GFP fluorescence in cells of Streptococcus gordonii under conditions of low $\mathrm{pH}$ and low oxygen concentration. Microbiology 147, 1383-1391.

Heidorn, T., Camsund, D., Huang, H. H., Lindberg, P., Oliveira, P., Stensjo, K., et al. (2011). Synthetic biology in cyanobacteria engineering and analyzing novel functions. Meth. Enzymol. 497, 539-579. doi: 10.1016/B978-0-12-385075$1.00024-\mathrm{X}$
Heyes, D. J., and Hunter, C. N. (2005). Making light work of enzyme catalysis: protochlorophyllide oxidoreductase. Trends Biochem. Sci. 30, 642-649. doi: 10.1016/j.tibs.2005.09.001

Higa, K. C., and Callahan, S. M. (2010). Ectopic expression of hetP can partially bypass the need for hetR in heterocyst differentiation by Anabaena sp. strain PCC 7120. Mol. Microbiol. 77, 562-574. doi: 10.1111/j.1365-2958. 2010.07257.x

Hilson, N., Rosengarten, R., and Keasling, J. (2012). j5 DNA assembly design automation software. ACS Synth. Biol. 1, 14-21. doi: 10.1021/sb2000116

Holtman, C., Chen, Y., Sandoval, P., Gonzales, A., Nalty, M., Thomas, T. et al. (2005). High-throughput functional analysis of the Synechococcus elongatus PCC 7942 Genome. DNA Res. 12, 103-115. doi: 10.1093/dnares/12.2.103

Huang, H. H., Camsund, D., Lindblad, P., and Heidorn, T. (2010). Design and characterization of molecular tools for a Synthetic Biology approach towards developing cyanobacterial biotechnology. Nucleic Acids Res. 38, 2577-2593. doi: 10.1093/nar/gkq164

Huang, H. H., and Lindblad, P. (2013). Wide-dynamic-range promoters engineered for cyanobacteria. J. Biol. Eng. 7:10. doi: 10.1186/1754-1611-7-10

Imamura, S., and Asayama, M. (2009). Sigma factors for cyanobacterial transcription. Gene Regul. Syst. Bio. 3, 65-87.

Jones, K. L., Kim, S. W., and Keasling, J. D. (2000). Low-copy plasmids can perform as well as or better than high-copy plasmids for metabolic engineering of bacteria. Metab. Eng. 2, 328-338. doi: 10.1006/mben.2000.0161

Kaczmarzyk, D., and Fulda, M. (2010). Fatty acid activation in cyanobacteria mediated by acylacyl carrier protein synthetase enables fatty acid recycling. Plant Physiol. 152, 1598-1610. doi: 10.1104/pp.109.148007

Knoop, H., Zilliges, Y., Lockau, W., and Steuer, R. (2010). The metabolic network of Synechocystis sp. PCC 6803: systemic properties of autotrophic growth. Plant Physiol. 154, 410-422. doi: 10.1104/pp.110.157198

Kopecna, J., Sobotka, R., and Komenda, J. (2013). Inhibition of chlorophyll biosynthesis at the protochlorophyllide reduction step results in the parallel depletion of Photosystem 
I and Photosystem II in the cyanobacterium Synechocystis PCC 6803. Planta 237, 497-508. doi: 10.1007/s00425-012-1761-4

Kumar, V. S., and Maranas, C. D. (2009). GrowMatch: an automated method for reconciling in silico/in vivo growth predictions. PLoS Comput. Biol. 5:e1000308. doi: 10.1371/journal.pcbi. 1000308

Kunert, A., Vinnemeier, J., Erdmann, N., and Hagemann, M. (2003). Repression by Fur is not the main mechanism controlling the iron-inducible isiAB operon in the cyanobacterium Synechocystis sp. PCC 6803. FEMS Microbiol. Lett. 227, 255-262. doi: 10.1016/S0378-1097(03)00689-X

Lagarde, D., Beuf, L., and Vermaas, W. (2000). Increased production of zeaxanthin and other pigments by application of genetic engineering techniques to Synechocystis sp. Strain PCC 6803. Appl. Environ. Microbiol. 66, 64-72. doi: 10.1128/AEM.66.1.64-72.2000

Landry, B., Stockel, J., and Pakrasi, H. (2012). Use of degradation tags to control protein levels in the cyanobacterium Synechocystis sp. Strain PCC 6803. Appl. Environ. Microbiol. 70, 2833-2835.

Latysheva, N., Junker, V. L., Palmer, W. J., Codd, G. A., and Barker, D. (2012). The evolution of nitrogen fixation in cyanobacteria. Bioinformatics 28, 603-606. doi: 10.1093/bioinformatics/bts008

Li, M. Z., and Elledge, S. J. (2007). Harnessing homologous recombination in vitro to generate recombinant DNA via SLIC. Nat. Methods 4, 251-256. doi: 10.1038/nmeth1010

Lindberg, P., Park, S., and Melis, A. (2010). Engineering a platform for photosynthetic isoprene production in cyanobacteria, using Synechocystis as the model organism. Metab. Eng. 12, 70-79. doi: 10.1016/j.ymben.2009.10.001

Liu, X., Sheng, J., and Curtiss, R. 3rd. (2011). Fatty acid production in genetically modified cyanobacteria. Proc. Natl. Acad. Sci. U.S.A. 108, 6899-6904. doi: 10.1073/pnas.1103014108

Mackey, S. R., Ditty, J. L., Clerico, E. M., and Golden, S. S. (2007). Detection of rhythmic bioluminescence from luciferase reporters in cyanobacteria. Methods Mol. Biol. 362, 115-129. doi: 10.1007/978-159745-257-1_8

Mahadevan, R., Edwards, J. S., and Doyle, F. J. (2002). Dynamic flux balance analysis of diauxic growth in Escherichia coli.
Biophys. J. 83, 1331-1340. doi: 10.1016/S0006-3495(02)73903-9

Mahadevan, R., and Schilling, C. H. (2003). The effects of alternate optimal solutions in constraintbased genome-scale metabolic models. Metab. Eng. 5, 264-276. doi: 10.1016/j.ymben.2003.09.002

Meighen, E. A. (1993). Bacterial bioluminescence: organization, regulation, and application of the lux genes. FASEB J. 7, 1016-1022.

Melis, A. (2013). Carbon partitioning in photosynthesis. Curr. Opin. Chem. Biol. 17, 453-456. doi: 10.1016/j.cbpa.2013.03.010

Michel, K. P., Pistorius, E. K., and Golden, S. S. (2001). Unusual regulatory elements for iron deficiency induction of the idiA gene of Synechococcus elongatus PCC 7942. J. Bacteriol. 183, 5015-5024. doi: 10.1128/JB.183.17. 5015-5024.2001

Mitschke, J., Georg, J., Scholz, I., Sharma, C. M., Dienst, D. Bantscheff, J., et al. (2011) An experimentally anchored map of transcriptional start sites in the model cyanobacterium Synechocystis sp. PCC6803. Proc. Natl. Acad. Sci. U.S.A. 108， 2124-2129. doi: 10.1073/pnas.1015154108

Montagud, A., Zelezniak, A., Navarro, E., De Cordoba, P., Urchueguia, J. F., and Patil, K. R. (2011). Flux coupling and transcriptional regulation within the metabolic network of the photosynthetic bacterium Synechocystis sp PCC6803. Biotechnol. J. 6, 330-342. doi: 10.1002/biot.201000109

Montgomery, B. L. (2007). Sensing the light: photoreceptive systems and signal transduction in cyanobacteria. Mol. Microbiol. 64, 16-27. doi: 10.1111/j.1365-2958.2007.05622.x

Moon, T. S., Lou, C., Tamsir, A., Stanton, B. C., and Voigt, C. A. (2012). Genetic programs constructed from layered logic gates in single cells. Nature 491, 249-253. doi: 10.1038/nature11516

Mukherjee, A., Weyant, K. B., Walker, J., and Schroeder, C. M. (2012). Directed evolution of bright mutants of an oxygen-independent flavinbinding fluorescent protein from Pseudomonas putida. J. Biol. Eng. 6, 20. doi: 10.1186/1754-1611-6-20

Nagarajan, A., Winter, R., Eaton-Rye, J., and Burnap, R. (2011). A synthetic DNA and fusion PCR approach to the ectopic expression of high levels of the D1 protein of photosystem II in Synechocystis sp. PCC 6803. J. Photochem.Photobiol.
B 104, 212-219. doi: 10.1016/j.jphotobiol.2011.02.009

Nakajima, M., Imai, K., Ito, $\mathrm{H}$. Nishiwaki, T., Murayama, Y., Iwasaki, H., et al. (2005) Reconstitution of circadian oscillation of cyanobacterial KaiC phosphorylation in vitro. Science 308, 414-415. doi 10.1126/science.1108451

Nakamura, Y., Kaneko, T., Miyajima, N., and Tabata, S. (1999). Extension of CyanoBase. CyanoMutants: repository of mutant information on Synechocystis sp. strain PCC6803. Nucleic Acids Res. 27, 66-68. doi: 10.1093/nar/27.1.66

Nakao, M., Okamoto, S., Kohara, M., Fujishiro, T., Fujisawa, T., Sato, S., et al. (2010). CyanoBase: the cyanobacteria genome database update 2010. Nucleic Acids Res. 38, D379-D381. doi 10.1093/nar/gkp915

Ng, W. O., Zentella, R., Wang, Y., Taylor, J. S., and Pakrasi, H. B. (2000). PhrA, the major photoreactivating factor in the cyanobacterium Synechocystis sp. strain PCC 6803 codes for a cyclobutane-pyrimidine-dimerspecific DNA photolyase. Arch Microbiol. 173, 412-417. doi: $10.1007 / \mathrm{s} 002030000164$

Niederholtmeyer, H., Wolfstadter, B. T., Savage, D. F., Silver, P. A., and Way, J. C. (2010). Engineering cyanobacteria to synthesize and export hydrophilic products. Appl. Environ. Microbiol. 76, 3462-3466. doi: 10.1128/AEM.00202-10

Nogales, J., Gudmundsson, S., Knight, E. M., Palsson, B. O., and Thiele, I. (2012). Detailing the optimality of photosynthesis in cyanobacteria through systems biology analysis. Proc. Natl. Acad. Sci. U.S.A. $\quad 109, \quad 2678-2683 . \quad$ doi: 10.1073/pnas.1117907109

Oliver, J. W., Machado, I. M., Yoneda, H., and Atsumi, S. (2013). Cyanobacterial conversion of carbon dioxide to 2,3-butanediol. Proc. Natl. Acad. Sci. U.S.A. 110, 1249-1254. doi: 10.1073/pnas.1213024110

Omata, T., Price, G. D., Badger, M. R., Okamura, M., Gohta, S., and Ogawa, T. (1999). Identification of an ATP-binding cassette transporter involved in bicarbonate uptake in the cyanobacterium Synechococcus sp. strain PCC 7942. Proc. Natl. Acad. Sci. U.S.A. 96, 13571-13576. doi: $10.1073 /$ pnas.96.23.13571

Orth, J. D., Thiele, I., and Palsson, B. O. (2010). What is flux balance analysis? Nat. Biotechnol. 28, 245-248. doi: 10.1038/nbt.1614
Peca, L., Kos, P. B., Mate, Z., Farsang, A., and Vass, I. (2008). Construction of bioluminescent cyanobacterial reporter strains for detection of nickel, cobalt and zinc. FEMS Microbiol. Lett. 289, 258-264. doi: 10.1111/j.1574-6968.2008.01393.x

Peca, L., Kos, P. B., and Vass, I. (2007). Characterization of the activity of heavy metal-responsive promoters in the cyanobacterium Synechocystis PCC 6803. Acta. Biol. Hung. 58, 11-22. doi: 10.1556/ABiol.58.2007.Suppl.2

Pfreundt, U., Stal, L. J., Voss, B., and Hess, W. R. (2012). Dinitrogen fixation in a unicellular chlorophyll d-containing cyanobacterium. ISME J. 6, 1367-1377. doi: 10.1038/ismej.2011.199

Qi, Q., Hao, M., Ng, W. O., Slater, S. C., Baszis, S. R., Weiss, J. D., et al. (2005). Application of the Synechococcus nirA promoter to establish an inducible expression system for engineering the Synechocystis tocopherol pathway. Appl. Environ. Microbiol. 71, 5678-5684. doi: $\quad$ 10.1128/AEM.71.10.56785684.2005

Quan, J., and Tian, J. (2009). Circular polymerase extension cloning of complex gene libraries and pathways. PLoS ONE 4:e6441. doi 10.1371/journal.pone.0006441

Saha, R., Verseput, A. T., Berla, B. M., Mueller, T. J., Pakrasi, H. B., and Maranas, C. D. (2012). Reconstruction and comparison of the metabolic potential of cyanobacteria Cyanothece sp. ATCC 51142 and Synechocystis sp. PCC 6803. PLOS ONE 7:e48285. doi: 10.1371/journal.pone.0048285

Sakuragi, Y. (2004). Studies of Quinones in Cyanobacteria. University Park, PA: Doctor of Philosophy; The Pennsylvania State University.

Satya Lakshmi, O., and Rao, N. M. (2009). Evolving Lac repressor for enhanced inducibility. Protein Eng. Des. Sel. 22, 53-58. doi: 10.1093/protein/gzn069

Savinell, J. M., and Palsson, B. O. (1992). Network analysis of intermediary metabolism using linear optimization. I. Development of mathematical formalism. J. Theor. Biol. 154, 421-454. doi: 10.1016/S0022-5193(05)80161-4

Schwarz, D., Orf, I., Kopka, J., and Hagemann, M. (2013). Recent applications of metabolomics toward cyanobacteria. Metabolites 3, 72-100. doi: 10.3390/metabo3010072

Selinger, D. W., Cheung, K. J., Mei, R., Johansson, E. M., Richmond, 
C. S., Blattner, F. R., et al. (2000). RNA expression analysis using a 30 base pair resolution Escherichia coli genome array. Nat. Biotechnol. 18, 1262-1268. doi: 10.1038/82367

Shao, Z., and Zhao, H. (2009). DNA assembler, an in vivo genetic method for rapid construction of biochemical pathways. Nucleic Acids Res. 37:e16. doi: 10.1093/nar/gkn991

Sharma, C. M., Hoffmann, S., Darfeuille, F., Reignier, J., Findeiss, S., Sittka, A., et al. (2010). The primary transcriptome of the major human pathogen Helicobacter pylori. Nature 464, 250-255. doi: 10.1038 /nature 08756

Shastri, A. A., and Morgan, J. A. (2005). Flux balance analysis of photoautotrophic metabolism. Biotechnol. Prog. 21, 1617-1626. doi: 10.1021/bp050246d

Simkovsky, R., Daniels, E. F., Tang, K., Huynh, S. C., Golden, S. S., and Brahamsha, B. (2012). Impairment of O-antigen production confers resistance to grazing in a model amoeba-cyanobacterium predatorprey system. Proc. Natl. Acad. Sci. U.S.A. 109, 16678-16683. doi: 10.1073/pnas.1214904109

Stockel, J., Jacobs, J. M., Elvitigala, T. R., Liberton, M., Welsh, E. A., Polpitiya, A. D., et al. (2011). Diurnal rhythms result in significant changes in the cellular protein complement in the cyanobacterium Cyanothece 51142. PLoS ONE 6:e16680. doi: 10.1371/journal.pone.0016680

Stockel, J., Welsh, E. A., Liberton, M., Kunnvakkam, R., Aurora, R., and Pakrasi, H. B. (2008). Global transcriptomic analysis of Cyanothece 51142 reveals robust diurnal oscillation of central metabolic processes. Proc. Natl. Acad. Sci. U.S.A. 105, 6156-6161. doi: 10.1073/pnas. 0711068105

Szewczyk, E., Nayak, T., Oakley, C. E., Edgerton, H., Xiong, Y., TaheriTalesh, N., et al. (2007). Fusion PCR and gene targeting in Aspergillus nidulans. Nat. Protoc. 1, 3111-3120. doi: 10.1038/nprot.2006.405

Takahama, K., Matsuoka, M., Nagahama, K., and Ogawa, T. (2004). High-frequency gene replacement in cyanobacteria using a heterologous rps12 gene. Plant Cell Physiol. 45, 333-339. doi: 10.1093/pcp/pch041

Tan, X., Liang, F., Cai, K., and Lu, X. (2013). Application of the FLP/FRT recombination system in cyanobacteria for construction of markerless mutants. Appl. Microbiol. Biotechnol. 97, 6373-6382. doi: 10.1007/s00253-013-4837-6
Taniuchi, Y., Yoshikawa, S., Maeda, S., Omata, T., and Ohki, K. (2008). Diazotrophy under continuous light in a marine unicellular diazotrophic cyanobacterium, Gloeothece sp. 68DGA. Microbiology 154, 1859-1865. doi: 10.1099/mic.0.2008/018689-0

Taton, A., Lis, E., Adin, D. M., Dong, G., Cookson, S., Kay, S. A., et al. (2012). Gene transfer in Leptolyngbya sp. strain BL0902, a cyanobacterium suitable for production of biomass and bioproducts. PLoS ONE 7:e30901. doi: 10.1371/journal.pone.0030901

Teng, S. W., Mukherji, S., Moffitt, J. R., De Buyl, S., and O'shea, E. K. (2013). Robust circadian oscillations in growing cyanobacteria require transcriptional feedback. Science 340, 737-740. doi: 10.1126/science. 1230996

Thiele, I., and Palsson, B. O. (2010). A protocol for generating a high-quality genome-scale metabolic reconstruction. Nat. Protoc. 5, 93-121. doi: 10.1038/ nprot.2009.203

Tyo, K. E., Jin, Y. S., Espinoza, F. A., and Stephanopoulos, G. (2009). Identification of gene disruptions for increased poly-3hydroxybutyrate accumulation in Synechocystis PCC 6803. Biotechnol. Prog. 25, 1236-1243. doi: 10.1002/btpr.228

Varma, A., Boesch, B. W., and Palsson, B. O. (1993). Stoichiometric interpretation of Escherichia coli glucose catabolism under various oxygenation rates. Appl. Environ. Microbiol. 59, 2465-2473.

Varma, A., and Palsson, B. O. (1994). Stoichiometric flux balance models quantitatively predict growth and metabolic by-product secretion in wild-type Escherichia coli W3110. Appl. Environ. Microbiol. 60, 3724-3731.

Von Berlepsch, S., Kunz, H. H., Brodesser, S., Fink, P., Marin, K., Flugge, U. I., et al. (2012). The acyl-acyl carrier protein synthetase from Synechocystis sp. PCC 6803 mediates fatty acid import. Plant Physiol. 159, 606-617. doi: 10.1104/pp.112.195263

Vu, T. T., Stolyar, S. M., Pinchuk, G. E., Hill, E. A., Kucek, L. A., Brown, R. N., et al. (2012). Genomescale modeling of light-driven reductant partitioning and carbon fluxes in diazotrophic unicellular cyanobacterium Cyanothece sp. ATCC 51142. PLoS Comput. Biol. 8:e1002460. doi: 10.1371/journal. pcbi. 1002460
Wang, B., Pugh, S., Nielsen, D. R., Zhang, W., and Meldrum, D. R. (2013). Engineering cyanobacteria for photosynthetic production of 3-hydroxybutyrate directly from $\mathrm{CO}_{2}$. Metab. Eng. 16C, 68-77. doi: 10.1016/j.ymben.2013.01.001

Wang, B., Wang, J., Zhang, W., and Meldrum, D. R. (2012). Application of synthetic biology in cyanobacteria and algae. Front. Microbiol. 3:344. doi: 10.3389/fmicb.2012.00344

Waters, C. M., and Bassler, B. L. (2006). The Vibrio harveyi quorum-sensing system uses shared regulatory components to discriminate between multiple autoinducers. Genes Dev. 20, 2754-2767. doi: 10.1101/gad.1466506

Welsh, E. A., Liberton, M., Stockel, J., Loh, T., Elvitigala, T., Wang, C., et al. (2008). The genome of Cyanothece 51142, a unicellular diazotrophic cyanobacterium important in the marine nitrogen cycle. Proc. Natl. Acad. Sci. U.S.A. 105, 15094-15099. doi: 10.1073/pnas.0805418105

Woelfle, M. A., Ouyang, Y., Phanvijhitsiri, K., and Johnson, C. H. (2004). The adaptive value of circadian clocks: an experimental assessment in cyanobacteria. Curr. Biol. 14, 1481-1486. doi: 10.1016/j.cub.2004.08.023

Wu, B., Zhang, B. C., Feng, X. Y., Rubens, J. R., Huang, R., Hicks, L. M., et al. (2010). Alternative isoleucine synthesis pathway in cyanobacterial species. Microbiology 156, 596-602. doi: 10.1099/mic.0.031799-0

Xu, Y., Alvey, R. M., Byrne, P. O. Graham, J. E., Shen, G., and Bryant, D. A. (2011). Expression of genes in cyanobacteria: adaptation of endogenous plasmids as platforms for high-level gene expression in Synechococcus sp. PCC 7002. Methods Mol. Biol. 684, 273-293. doi: 10.1007/978-1-60761-925-3_21

Xu, Y., Ma, P., Shah, P., Rokas, A., Liu, Y., and Johnson, C. H. (2013). Nonoptimal codon usage is a mechanism to achieve circadian clock conditionality. Nature 495, 116-120. doi: 10.1038/nature11942

Yang, C., Hua, Q., and Shimizu, K. (2002). Metabolic flux analysis in Synechocystis using isotope distribution from 13Clabeled glucose. Metab. Eng. 4, 202-216. doi: 10.1006/mben. 2002.0226

Young, J. D., Shastri, A. A., Stephanopoulos, G., and Morgan, J. A. (2011). Mapping photoautotrophic metabolism with isotopically nonstationary (13)C flux analysis. Metab. Eng. 13, 656-665. doi: 10.1016/j.ymben.2011.08.002

Zhang, F., Carothers, J. M., and Keasling, J. D. (2012). Design of a dynamic sensor-regulator system for production of chemicals and fuels derived from fatty acids. Nat. Biotechnol. 30, 354-359. doi: 10.1038/nbt.2149

Zhang, S. Y., and Bryant, D. A. (2011). The tricarboxylic acid cycle in cyanobacteria. Science 334, 1551-1553. doi: 10.1126/science. 1210858

Zhang, Y., Pu, H., Wang, Q., Cheng, S., Zhao, W., Zhang, Y., et al. (2007). PII is important in regulation of nitrogen metabolism but not required for heterocyst formation in the Cyanobacterium Anabaena sp. PCC 7120. J. Biol. Chem. 282, 33641-33648. doi: 10.1074/jbc.M706500200

Zomorrodi, A. R., Suthers, P. F., Ranganathan, S., and Maranas, C. D. (2012). Mathematical optimization applications in metabolic networks. Metab. Eng. 14, 672-686. doi: 10.1016/j.ymben. 2012.09.005

Conflict of Interest Statement: The authors declare that the research was conducted in the absence of any commercial or financial relationships that could be construed as a potential conflict of interest.

Received: 14 June 2013; accepted: 05 August 2013; published online: 27 August 2013.

Citation: Berla BM, Saha R, Immethun CM, Maranas CD, Moon TS and Pakrasi HB (2013) Synthetic biology of cyanobacteria: unique challenges and opportunities. Front. Microbiol. 4:246. doi: 10.3389/fmicb.2013.00246

This article was submitted to Microbial Physiology and Metabolism, a section of the journal Frontiers in Microbiology.

Copyright (c) 2013 Berla, Saha, Immethun, Maranas, Moon and Pakrasi. This is an open-access article distributed under the terms of the Creative Commons Attribution License (CC BY). The use, distribution or reproduction in other forums is permitted, provided the original author(s) or licensor are credited and that the original publication in this journal is cited, in accordance with accepted academic practice. No use, distribution or reproduction is permitted which does not comply with these terms. 